hep-th/9811214

YCTP-P27-98

\title{
3-manifold topology and the Donaldson-Witten partition function
}

\author{
Marcos Mariño and Gregory Moore \\ Department of Physics, Yale University, \\ New Haven, CT 06520 \\ marcos.marino@yale.edu \\ moore@castalia.physics.yale.edu
}

\begin{abstract}
We consider Donaldson-Witten theory on four-manifolds of the form $X=Y \times \mathbf{S}^{1}$ where $Y$ is a compact three-manifold. We show that there are interesting relations between the four-dimensional Donaldson invariants of $X$ and certain topological invariants of $Y$. In particular, we reinterpret a result of Meng-Taubes relating the Seiberg-Witten invariants to Reidemeister-Milnor torsion. If $b_{1}(Y)>1$ we show that the partition function reduces to the Casson-Walker-Lescop invariant of $Y$, as expected on formal grounds. In the case $b_{1}(Y)=1$ there is a correction. Consequently, in the case $b_{1}(Y)=1$, we observe an interesting subtlety in the standard expectations of Kaluza-Klein theory when applied to supersymmetric gauge theory compactified on a circle of small radius.
\end{abstract}

Nov. 23, 1998 


\section{Introduction}

The application of gauge theory to the topology of 3- and 4-manifolds has proved to be a deep and rich subject. This subject is now mature and has an extensive literature. Nevertheless, important unsolved problems and challenges remain. Many of these open problems are related to Floer homology, and the topology of three-manifolds.

Last year, there was some progress in the application of Witten's physical approach to Donaldson theory via supersymmetric gauge theory [1] [2] [3]. This progress was the result of an improved understanding of $\mathcal{N}=2$ supersymmetric Yang-Mills theory on 4manifolds $X$ with $b_{2}^{+}(X)=1$ [4]. For example, the extension of Donaldson-Witten theory to nonsimply connected manifolds was recently completed in [5].

In the present paper we continue to explore some of the consequences of the results of [5], focusing on the applications to 3-manifold topology. In particular, we discuss the evaluation of the Donaldson-Witten function $Z_{D W}\left(Y \times \mathbf{S}^{1}\right)$ for 3-manifolds $Y$ with $b_{1}(Y)>$ 0 . The function $Z_{D W}(X)$ for a four-manifold $X$ is the partition function of a topologicallytwisted $d=4, \mathcal{N}=2$ gauge theory on $X$. In this paper we concentrate on the gauge groups $S U(2)$ and $S O(3)$, although generalizations should be accessible using [6].

The supersymmetric Yang-Mills approach is particularly well-suited to addressing questions related to Floer homology since the Floer groups are the Hilbert spaces of quantum ground states of the topological field theory [1]. Given a 3-fold $Y$ the path integral $Z_{D W}\left(Y \times \mathbf{S}^{1}\right)$ is just the Witten index $\operatorname{Tr}(-1)^{\mathrm{F}}$ of the theory and therefore is the Euler character of the Floer homology. The arguments for this are of course formal, and we check the result for $b_{1}(Y)>1$ in section 4.2 .

In section five we move on to the more difficult case of $b_{1}(Y)=1$. In this case we encounter an interesting subtlety in the compactification of supersymmetric Yang-Mills theory on a circle. This subtlety is connected with the noncompact nature of the moduli space of SYM groundstates. We consider a product metric on $X=Y \times \mathbf{S}^{1}$ where the $\mathbf{S}^{1}$ factor has radius $R$. Taking the $R \rightarrow 0$ limit one finds an effective 3 -dimensional theory described in detail in [7]. When topologically twisted, the partition function of this theory defines the Rozansky-Witten invariant of $Y$ [8]. On the other hand we may evaluate $Z_{D W}\left(Y \times \mathbf{S}^{1}\right)$ directly and take the $R \rightarrow 0$ limit. For 3-folds $Y$ with $b_{1}(Y)=1$ the answer does not agree with the Rozansky-Witten invariant. Thus, the naive Kaluza-Klein expectation fails for this class of observables. We discuss some of the physics associated with this surprising fact in section 6. Our main conclusion is that the theory must be simultaneously regarded as three- and four-dimensional. 


\section{A brief review of some three-manifold invariants}

We will need some basic facts and results about Reidemeister-Milnor torsion and Alexander polynomials of three manifolds as well as some related results on knot and link invariants. We review these for the reader's convenience. Good references for these results are [9] 10.11].

Let $Y$ be a connected, oriented three-dimensional manifold with $b_{1}(Y)>0$. The situations we will have in mind are compact manifolds or the complement of a link in a compact manifold. The invariants of $Y$ depend in part on the structure of the first homology group of $Y, H_{1}(Y, \mathbb{Z})$. This is a finitely generated abelian group. Accordingly it has a free part and a torsion part:

$$
H_{1}(Y, \mathbb{Z})=\mathbb{Z}^{b_{1}(Y)} \oplus \operatorname{Tor}\left(H_{1}(Y, \mathbb{Z})\right)
$$

where $\operatorname{Tor}\left(H_{1}(Y, \mathbb{Z})\right)$ is the subgroup of elements of finite order. We denote the associated free abelian group of rank $b_{1}(Y)$ by

$$
H(Y) \equiv H_{1}(Y, \mathbb{Z}) / \operatorname{Tor}\left(H_{1}(Y, \mathbb{Z})\right) \cong \mathbb{Z}^{b_{1}(Y)} .
$$

Note that, by the universal coefficient theorem, $H(Y) \simeq H^{2}(Y, \mathbb{Z}) / \operatorname{Tor}\left(H^{2}(Y, \mathbb{Z})\right)$. Here and below we will sometimes simply write $H$ when no confusion can arise. We will also denote the generators of $H$ by $h_{1}, \ldots, h_{b_{1}}$. By Poincaré duality, this gives a basis for the free part of $H^{2}(Y, \mathbb{Z})$ that will be denoted by $h_{i}$ as well, with $i=1, \ldots, b_{1} \equiv b_{1}(Y)$.

The invariants of $Y$ that we will discuss are multivariable Laurent polynomials and Laurent series in variables $t_{i}$ which can be formally identified as $t_{i}=\exp \left(h_{i}\right)$. (We are being somewhat imprecise here for the sake of expediency. Fussbudgets should consult the remark at the end of this section.) The Reidemeister-Milnor torsion of $Y$, which we will denote by $\tau\left(Y ; t_{i}\right)$, is in general a formal power series in $t_{i}, t_{i}^{-1}$. The precise definition of this invariant, (which we will not need) can be found in [9]. Roughly speaking, one introduces a combinatorial description of $Y$ and considers ratios of volumes of the terms in the associated (acyclic) chain complexes.

One of the basic properties of the torsion is that it is symmetric under the exchange $t_{i} \leftrightarrow t_{i}^{-1}$. The Reidemeister-Milnor torsion of $Y$ is related to the analytic Ray-Singer torsion, which is defined in terms of the Laplacian operator on forms with coefficient in a flat bundle. For instance, if $b_{1}(Y)=1$, and we consider the flat $U(1)$ connection $A_{\mu}=u \omega_{\mu}$, where $\omega$ is a harmonic one form that represents a generator of $H^{1}(Y, \mathbb{R})$, then $\left|\tau\left(Y ; \mathrm{e}^{i u}\right)\right|$ is 
the Ray-Singer torsion associated to the flat connection. Similar considerations apply when $b_{1}(Y)>1$. This equivalence is important in the analysis of perturbative Chern-Simons theory [12][13].

Another classical invariant of three manifolds is the Alexander polynomial of $Y$, denoted by $\Delta_{Y}\left(t_{i}\right)$, which is a Laurent polynomial in the $t_{i}$. The Reidemeister-Milnor torsion is related to the Alexander polynomial as follows (see [9], Theorem 1.1.2): if $b_{1}(Y)>1$, one has

$$
\tau\left(Y ; t_{i}\right)=\Delta_{Y}\left(t_{i}\right), \quad b_{1}(Y)>1
$$

In particular, for $b_{1}(Y)>1$, the Reidemeister-Milnor torsion is a Laurent polynomial.

On the other hand, if $b_{1}(Y)=1$, then

$$
\tau(Y ; t)=\frac{\Delta_{Y}(t)}{\left(t^{1 / 2}-t^{-1 / 2}\right)^{\epsilon(Y)}}, \quad b_{1}(Y)=1
$$

where $\epsilon(Y)=2$ if $Y$ is closed and 1 if $Y$ has a boundary. 1 Note that for $b_{1}(Y)=1$ the torsion $\tau(Y)$ is an infinite Laurent series. For $b_{1}(Y)=1$ the Alexander polynomial is also symmetric under $t \rightarrow t^{-1}$ with the structure

$$
\Delta_{Y}(t)=a_{0}+\sum_{i=1}^{r} a_{i}\left(t^{i}+t^{-i}\right)
$$

Moreover, it satisfies the important property ([9], Theorem 1.6.1)

$$
\Delta_{Y}(1)=\left|\operatorname{Tor}\left(H_{1}(Y, \mathbb{Z})\right)\right|
$$

Using this result, the Reidemeister-Milnor torsion for a closed manifold $Y$ with $b_{1}(Y)=1$ can be rewritten in the form:

$$
\tau(Y ; t)=\frac{\left|\operatorname{Tor}\left(H_{1}(Y, \mathbb{Z})\right)\right|}{\left(t^{1 / 2}-t^{-1 / 2}\right)^{2}}+\sum_{j=1}^{r} j a_{j}+\sum_{k=1}^{r-1}\left[\left(\sum_{j=1}^{r-k} j a_{j+k}\right)\left(t^{k}+t^{-k}\right)\right]
$$

This representation will prove useful in some of the derivations below.

1 The definition of the torsion for manifolds with boundary requires some discussion of boundary conditions that we don't need here. 


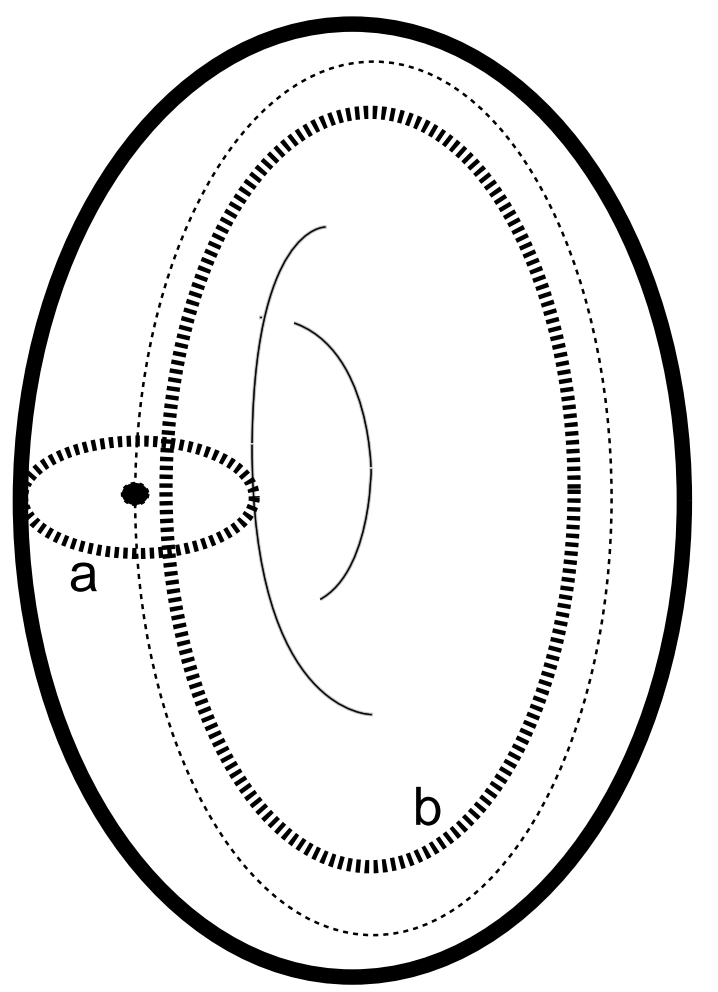

Fig. 1: The thin dashed line is the component of a link, surrounded by a solid torus. The two thick dashed lines are on the boundary of the torus. The longitude runs parallel to the link-component ("b-cycle") and the meridian runs horizontal ("a-cycle").

We now briefly consider invariants of links. We will consider links $\ell$ embedded in rational homology spheres $R$. Every knot in the link has a tubular neighborhood which is a solid torus. One of the one-cycles in the boundary of this solid torus is a parallel of the knot, while the other one-cycle is the meridian of the knot. The complement of a link with $n$ components in a rational homology sphere is a three-manifold with boundary $Y=R \backslash \ell$, and with $b_{1}(Y)=n$. The generators of the free abelian group $H$ are the meridians of the link components. The Reidemeister-Milnor torsion or Alexander polynomial of a link will be defined as the Reidemeister-Milnor torsion or Alexander polynomial of the manifold $Y$. In addition, one defines the multivariable, symmetric Conway function of a link $\ell \subset R$ as $⿴$

$$
\nabla_{\ell}\left(t_{1}, \ldots, t_{n}\right)=\tau\left(R \backslash \ell ; t_{1}, \ldots, t_{n}\right) .
$$

${ }^{2}$ For $n>1 \nabla_{\ell}$ is a Laurent polynomial. 
Finally, we will need the one-variable Conway polynomial $\nabla_{\ell}(z)$, where $z=t^{1 / 2}-$ $t^{-1 / 2}$. It is well known that this polynomial can be easily computed from a simple set of skein relations and is related to the Milnor torsion as follows (see [10], 2.3.13):

$$
\left|H_{1}(R, \mathbb{Z})\right| \nabla_{\ell}(z)=\left(t^{1 / 2}-t^{-1 / 2}\right) \tau(R \backslash \ell ; t, \ldots, t),
$$

where $\left|H_{1}(R, \mathbb{Z})\right|$ is the order of the finite group $H_{1}(R, \mathbb{Z})$. Notice, using (2.4), that if $R=\mathbf{S}^{3}$, the Alexander polynomial of a knot is equal to its Conway polynomial after the change of variable $z=t^{1 / 2}-t^{-1 / 2}$. In general, the Conway polynomial of an $n$-component link has the structure (see for instance [11], Proposition 8.7):

$$
\nabla_{\ell}(z)=z^{n-1}\left(a_{0}+a_{1} z^{2}+\ldots+a_{d} z^{2 d}\right) .
$$

Remark. In the above discussion we were somewhat imprecise about the nature of the Laurent series $\tau\left(Y ; t_{i}\right)$. The precise story is the following. We can form two rings starting from $H, \mathbb{Z}[H]$ and $\mathbb{Z}[[H]]$. The elements of $\mathbb{Z}[H]$ are the Laurent polynomials $\mathbb{Z}\left[t_{1}, t_{1}^{-1}, \ldots, t_{b_{1}}, t_{b_{1}}^{-1}\right]$, where formally $t_{i}=\exp \left(h_{i}\right) . \mathbb{Z}[H]$ is called the integral group ring of $H$, and can be understood as the set of finitely supported $\mathbb{Z}$-valued functions on $H$. We can introduce a multiplicative action of $H$ on $\mathbb{Z}[H]$ as follows: if $h \in H$ is written as $h=\sum n_{i} h_{i}$, where $n_{i} \in \mathbb{Z}$, and $f\left(t_{1}, \ldots, t_{b_{1}}\right) \in \mathbb{Z}[H]$, then $h \cdot f\left(t_{1}, \ldots, t_{b_{1}}\right)=t_{1}^{n_{1}} \ldots t_{b_{1}}^{n_{b_{1}}} f\left(t_{1}, \ldots, t_{b_{1}}\right)$. The orbits of this action are $\mathbb{Z}[H] / H$, and the elements of this quotient group are elements of $\mathbb{Z}[H]$ defined up to multiplication by an element of the form $t_{1}^{n_{1}} \ldots t_{b_{1}}^{n_{b_{1}}}$. The reason to define this quotient group is that the invariants we will consider, namely the Alexander polynomial and the torsion of a three-manifold, are usually defined up to multiplication by an element of this form (this is already the case, for example, for the Alexander polynomial of a knot; see for instance [11].) Notice that there is a natural conjugation operation on $\mathbb{Z}[H]$ given by $t_{i} \leftrightarrow t_{i}^{-1}$. The elements of $\mathbb{Z}[[H]]$ can be understood as formal series in the variables $t_{i}, t_{i}^{-1}$, therefore $\mathbb{Z}[[H]]$ is the set of $\mathbb{Z}$-valued functions on $H$ (not necessarily with a finite support). It can also be identified to the field of fractions of $\mathbb{Z}[H]$. The Reidemeister-Milnor torsion is an element in $\mathbb{Z}[[H]] / H$. It can be shown that it always has a symmetric representative under $t_{i} \leftrightarrow t_{i}^{-1}$, and throughout this paper we will always work with symmetric representatives. Similarly, the Alexander polynomials $\Delta_{Y}\left(t_{i}\right)$ is properly thought of as an element of $\mathbb{Z}[H] / H$. It too has a symmetric representative with which we will always work. 


\section{Seiberg-Witten invariants of $Y \times \mathbf{S}^{1}$}

In this section we will consider the SW invariants of four-manifolds with the structure $X=Y \times \mathbf{S}^{1}$, and $b_{1}(Y)>0$. We first show using simple arguments that the $\mathrm{SW}$ invariants of $X$ are determined by the three-dimensional SW invariants of $Y$. 3 Then we describe the relation of the three-dimensional SW invariants to the Reidemeister-Milnor torsion, using the results of Meng and Taubes 15.

\subsection{Seiberg-Witten invariants in three and four dimensions}

The Seiberg-Witten equations and invariants can be defined for both four-manifolds $X$ and three-manifolds $Y$. The SW monopole equations [3] involve a pair $(A, M)$ consisting of a connection and a section $M$ of a $\operatorname{Spin}^{c}$ line bundle $c$. These equations can be written schematically as:

$$
\begin{aligned}
& F(A)=\bar{M} \Gamma M \\
& \not D_{A} M=0 .
\end{aligned}
$$

In addition to the four-dimensional version, the three dimensional equations have been extensively studied over the past few years both from the point of view of mathematics [16] [17], and also from the point of view of quantum field theory [18]. The available $\operatorname{Spin}^{c}$ structures are determined as follows. Every three-dimensional manifold is a Spin manifold [19]. The $\operatorname{Spin}^{c}$ structures on $Y$ are simply $U(2)$ bundles $W_{c}$, with first Chern class $c_{1}(c) \in$

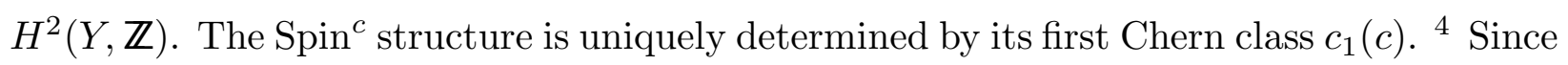
$Y$ is Spin, $c_{1}(c)$ is always divisible by 2 in $H^{2}(Y, \mathbb{Z})$. Here and below we will denote the projection of $c_{1}(c)$ into the free abelian group $H(Y)=H^{2}(Y, \mathbb{Z}) / \operatorname{Tor}\left(H^{2}(Y, \mathbb{Z})\right)$ by $\bar{c}_{1}(c)=$ $x$. As in four dimensions, one now defines the three-dimensional SW invariant, denoted by $S W(Y, c)$, as a signed sum of solutions modulo gauge equivalence to the equations (3.1).

Let us now consider the relation between the SW invariants on a three-manifold $Y$ and those on the associated four-manifold $X=Y \times \mathbf{S}^{1}$, where $Y$ is a compact, oriented three-manifold with $b_{1}(Y)>0$. The numerical invariants are related by $\chi(X)=\sigma(X)=0$, $b_{1}(X)=b_{1}(Y)+1$. We also have $b_{2}^{+}(X)=b_{1}(Y)$.

3 A more rigorous argument would follow the lines of [14, where a similar situation was analyzed involving the three-dimensional monopole equations on $\Sigma_{g} \times \mathbf{S}^{1}, \Sigma_{g}$ being a Riemann surface.

4 After we have fixed an origin for the action of the 2-torsion elements on the $\operatorname{Spin}^{c}$ structures. Technically, the set of $\operatorname{Spin}^{c}$ structures is a "torsor." 
As for the SW invariants we will now show that the $\operatorname{Spin}^{c}$ structures of $X$ and $Y$ supporting nonzero invariants are essentially the same. Since $Y$ is spin, so is $X$. The first thing to notice is that, even when $b_{1}(Y)=1$, there is no wall-crossing (WC) for the SW invariants of $X$, provided that they are computed with a small perturbation of the equations. (There is wall-crossing for the stable SW invariants computed with a very large perturbation, and this will be considered presently.) The reason for this is that non-trivial SW invariants arise only when the dimension of the moduli space associated to a $\operatorname{Spin}^{c}$ structure with determinant line bundle $2 \lambda$ is $d_{\lambda}=\lambda^{2} \geq 0$ (recall that $\sigma(X)=\chi(X)=0$ ), but for small perturbations of the SW equations a necessary condition for WC is $\lambda^{2}<0$. Therefore, we can choose any metric on $Y \times \mathbf{S}^{1}$ to compute the invariants. We will work with a product metric corresponding to a radius $R$ for the circle, i.e. $g=R^{2} d \varphi^{2}+h_{i j} d x^{i} d x^{j}$, where $\varphi, x^{i}$ are coordinates for the circle and $Y$, respectively, and $h_{i j}$ is an arbitrary Riemannian metric on $Y$. The four-dimensional Dirac equation equation $\Gamma^{\mu} D_{\mu} M=0$, $\mu=\varphi, i$ can be written in local coordinates as

$$
\frac{1}{R} \Gamma^{\varphi} D_{\varphi} M+\Gamma^{i} D_{i} M=0
$$

where $\left(\Gamma^{\varphi}\right)^{2}=1$. If we now consider the limit $R \rightarrow 0$, we see that the monopole field has to be covariantly constant in the direction of the circle. Similarly, the other monopole equation $F^{+}-\bar{M} \Gamma M=0$ implies in this limit that $F_{i \varphi}=0$. This means that the Chern class of the $\operatorname{Spin}^{c}$ structure is a two-cohomology class on $Y$, in other words, that the monopole equations will have a solution only if the $\operatorname{Spin}^{c}$-structure on $X$ is the pullback of a $\operatorname{Spin}^{c}$ structure on $Y$. Therefore, the pair $(A, M)$ is induced (up to a gauge transformation) by a pair $(A, M)$ on $Y$, and what we have to solve are precisely the monopole equations that arise on the three-dimensional manifold $Y$ by dimensional reduction.

One can in fact prove that the SW invariants for the manifold $X$ are given by [17

$$
S W\left(Y \times \mathbf{S}^{1}, \pi^{*}(c)\right)=S W(Y, c)
$$

where the $\operatorname{Spin}^{c}$-structure on $X$ is the pullback $\pi^{*}(c)$ of a $\operatorname{Spin}^{c}$-structure $c$ on $Y$. As we have seen, the other $\operatorname{Spin}^{c}$ structures on $X$ have a zero invariant. This means that the four-manifold $X=Y \times \mathbf{S}^{1}$ is of simple type, as the only possible basic classes satisfy $x^{2}=0$. 


\subsection{The Meng-Taubes result}

As we have seen, the problem of computing the SW invariants of the four-manifold $Y \times \mathbf{S}^{1}$ reduces to the problem of computing the $3 \mathrm{D} \mathrm{SW}$ invariants of the manifold $Y$. A very interesting structural result for these invariants has been found by Meng and Taubes [15].

\subsection{1. $b_{1}(Y)>1$}

First we describe the result for three-manifolds with $b_{1}(Y)>1$. We define the SeibergWitten polynomial $\underline{S W}\left(t_{i}\right) \in \mathbb{Z}[H] / H$ as follows. Let $x=\sum_{i=1}^{b_{1}} 2 k_{i} h_{i}$ be the free part of the first Chern class of a $\operatorname{Spin}^{c}$-structure $c$ on $Y$, where $k_{i}$ are integers and $h_{i}$ are generators of $H=H^{2}(Y, \mathbb{Z}) / \operatorname{Tor}\left(H^{2}(Y, \mathbb{Z})\right)$, and let $S W(Y, c)$ be the corresponding SW invariant. The SW series of $Y$ is defined as

$$
\underline{S W}\left(t_{i}\right)=\sum_{x \in H}\left(\sum_{c \mid \bar{c}_{1}(c)=x} S W(Y, c)\right) t_{1}^{k_{1}} \ldots t_{b_{1}}^{k_{b_{1}}}
$$

where, for a given $x$ in $H$, we sum over all the SW invariants of the $\left|\operatorname{Tor} H^{2}(Y, \mathbb{Z})\right| \operatorname{Spin}^{c}$ structures $c$ with the same $x$. A different choice of generators gives a different representative in the orbit. In the orbit of $\underline{S W}$ there is always an element which is invariant under conjugation. This is a consequence of the charge-conjugation invariance of the SW equations [3]. The main result of Meng-Taubes is that

$$
\underline{S W}\left(t_{i}\right)=\tau\left(Y ; t_{i}\right)
$$

where $\tau\left(Y ; t_{i}\right)$ is the Milnor torsion. In this case, both elements are in $\mathbb{Z}[H]$, i.e. they are polynomials in the $t_{i}, t_{i}^{-1}$ variables. On the SW side, this is a simple consequence of the fact that there are only a finite number of basic classes.

As a simple example of (3.5), one can consider the three-manifold $Y=\Sigma_{g} \times \mathbf{S}^{1}$, where $\Sigma_{g}$ is a Riemann surface of genus $g$. In this case, the torsion is $\tau\left(\Sigma_{g} \times \mathbf{S}^{1} ; t_{i}\right)=$ $\left(t_{1}^{1 / 2}-t_{1}^{-1 / 2}\right)^{2 g-2}$, where $t_{1}=\mathrm{e}^{2\left[\mathbf{S}^{1}\right]}$. This is precisely the $\mathrm{SW}$ series of the manifold $\Sigma_{g} \times \mathbf{T}^{2}$, and using (3.3) we see that (3.5) holds. 


\subsection{2. $b_{1}(Y)=1$}

We now consider the structure of the invariants when $b_{1}(Y)=1$. In this case, the SW invariants have a mild dependence on the perturbation of the equations, and this amounts to a choice of a generator of $H^{1}(Y, \mathbb{Z})\left[15\right.$. Notice that, as $H^{1}(Y, \mathbb{Z}) \simeq \mathbb{Z}$, there are only two possible generators that differ in their sign. 5 To understand this dependence on the perturbation, it is useful to consider a four-dimensional version of this story, focusing again on the four manifold $X=Y \times \mathbf{S}^{1}$, with $b_{1}(Y)=1$. The structure of the cohomology ring of $X$ is the following: We denote by $S_{2}$ a generator of $H_{2}(Y, \mathbb{Z})$ and by $\gamma$ a generator of $H_{1}(Y, \mathbb{Z}) / \operatorname{Tor}\left(H_{1}(Y, \mathbb{Z})\right)$. Then there is another two-cycle in $X$ given by cup product $S_{1}=\gamma \cup \mathbf{S}^{1}$, where $\mathbf{S}^{1}$ denotes here a generator of $H_{1}\left(\mathbf{S}^{1}\right)$. We also have cohomology classes $\alpha_{Y} \in H^{2}(Y, \mathbb{Z}) / \operatorname{Tor}\left(H^{2}(Y, \mathbb{Z})\right)$ and $\beta_{Y} \in H^{1}(Y, \mathbb{Z}) \simeq \mathbb{Z}$, and such that

$$
\left[S_{1}\right]=\alpha_{Y}, \quad\left[S_{2}\right]=\beta_{Y} \wedge e
$$

where $e=d \varphi$ is a generator of $H^{1}\left(\mathbf{S}^{1}, \mathbb{Z}\right)$ and the brackets mean Poincaré duals. The intersection form of $X$ is the even unimodular lattice $I I^{1,1}$, and $\left[S_{1}\right] \cdot\left[S_{2}\right]=1$.

If we consider the usual SW invariants (with a very small perturbation), there will be no wall-crossing, as we remarked above. Assume now that we turn on a perturbation, and we consider the "twisted" SW equations on $X$ [3] 20]

$$
(F+2 \pi i \beta)_{+}=\bar{M} M
$$

where $\beta$ is a closed two-form which represents $b \in H^{2}(X, \mathbb{R})$. Equivalently, we consider a Spin $^{c}$-structure such that the free part of its first Chern class is $x-b$.

There is now a chamber structure in the space of perturbations, where the walls are defined by

$$
x=b \text {. }
$$

We will choose the perturbation in such a way that $b=2 r \alpha_{Y}$, where $r$ is a real number. Following the arguments in the previous subsection, we again see that the four-dimensional SW invariants will be zero for $\operatorname{Spin}^{c}$-structures which are not the pullback of a $\operatorname{Spin}^{c}$ structure on $Y$. If the $\operatorname{Spin}^{c}$-structure is the pullback of a $\operatorname{Spin}^{c}$-structure $c$ on $Y$, with

\footnotetext{
${ }^{5}$ Note in particular that $H^{1}(Y, \mathbb{Z})$ has no torsion, in contrast to $H^{2}(Y, \mathbb{Z})$.
} 


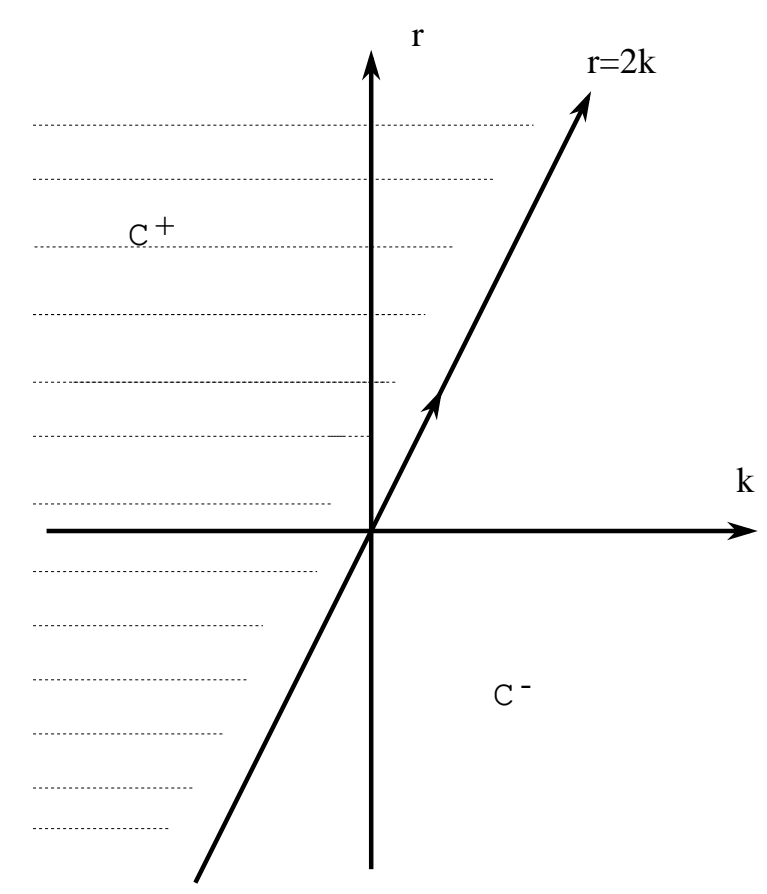

Fig. 2: Chamber structure in the space of perturbations and line bundles for the case $b_{1}(Y)=1$. The perturbation has cohomology class $b=2 r \alpha_{Y}$ and the line bundle has first Chern class $x=2 k \alpha_{Y}$. When $k>0$ the number of solutions of (3.7) jumps by $+k$ as we cross from chamber $\mathcal{C}^{-}$to $\mathcal{C}^{+}$.

$x$ of the form $x=2 k \alpha_{Y}$, the corresponding SW invariants will be the SW invariants computed from the perturbed $3 \mathrm{~d}$ monopole equation

$$
F(A)=\bar{M} \Gamma M-2 \pi i r \alpha_{Y}
$$

for the $\operatorname{Spin}^{c}$-structure $c$ on $Y$. As the walls are given by (3.8), the chambers are

$$
\mathcal{C}^{+}=\left\{b=r \alpha_{Y} \in H^{2}(Y, \mathbb{R}): r>2 k\right\}, \quad \mathcal{C}^{-}=\left\{b=r \alpha_{Y} \in H^{2}(Y, \mathbb{R}): r<2 k\right\} .
$$

and are illustrated in fig. 2 .

The perturbation in (3.7) has a very natural interpretation in terms of the underlying physical theory (at least in the Kähler case): when the twisted theory is perturbed by a mass term that breaks $\mathcal{N}=2$ supersymmetry down to $\mathcal{N}=1$, as in [2], the mass parameter must be a self-dual two-form . In the effective theory, this perturbation modifies the usual Seiberg-Witten monopole equations and gives (3.7).

We can now define the "stable" SW invariants $S W^{ \pm}(Y, c)$ in such a way that, for every Spin ${ }^{c}$-structure $c$, the SW invariant is computed in the chamber $\mathcal{C}^{ \pm}$, i.e. after turning on 
a perturbation with $r$ sufficiently large. It is easy to see that the choice of a chamber is equivalent to a choice of a generator for $H^{1}(Y, \mathbb{Z})[15]$. Let $o= \pm \beta_{Y}$ be a generator of $H^{1}(Y, \mathbb{Z})$. If we write the condition on the perturbation as 15

$$
\int_{Y} b \wedge o>\int_{Y} c_{1}(c) \wedge o
$$

we see that the choices $o= \pm \beta_{Y}$ give the two chambers $\mathcal{C}^{ \pm}$, i.e., $r>2 k$ and $r<2 k$. Using these stable invariants we can define a series just as in (3.4):

$$
\underline{S W^{ \pm}}=\sum_{x \in H}\left(\sum_{c \mid \bar{c}_{1}(c)=x} S W^{ \pm}(Y, c)\right) t^{k} .
$$

Notice that in the stable case there are infinitely many characteristic elements with a nonzero SW invariant. This is a consequence of the definition of stability, which involves an arbitrarily large perturbation of the equations. In fact, the effect of considering the stable invariants is to add an (infinite) universal series to the polynomial defined by the usual Seiberg-Witten invariants $S W(Y, c)$ (computed for a small perturbation). This can be seen as follows: let $c$ be a Spin ${ }^{c}$-structure with $x=\bar{c}_{1}(c)=2 k \alpha_{Y}$, and let's consider the stable SW invariants associated to the chamber $\mathcal{C}^{+}$. If $k<0$, a small perturbation is already in the chamber $\mathcal{C}^{+}$, so $S W^{+}(Y, c)=S W(Y, c)$. When $k>0$, the difference between the invariants is the wall-crossing term. The wall-crossing formula for a nonsimply connected four-manifold $X$ has been obtained in [20] [21], and rederived in [5] using the $u$-plane integral. In general, one has:

$$
S W^{+}(c)-S W^{-}(c)=\frac{1}{2}(-1)^{b_{1}}\left(c_{1}(c), \Sigma\right) \operatorname{vol}\left(\mathbf{T}^{b_{1}}\right)
$$

where $\Sigma$ is a certain two-dimensional cohomology class in $X$, and $\mathbf{T}^{b_{1}}$ is the torus $H_{1}(X, \mathbb{R}) / H$ (see [5] for the details). Notice that all the $\operatorname{Spin}^{c}$-structures with the same $x=\bar{c}_{1}(c)$ have the same wall-crossing behavior. In our case, the four-manifold $X=Y \times \mathbf{S}^{1}$ has $b_{1}(X)=2$, and the two-dimensional class is given by

$$
\Sigma=\beta_{Y} \wedge e=\left[S_{2}\right]
$$

while $x=2 k\left[S_{1}\right]$. Therefore, using (3.13), we obtain, for $k>0$,

$$
S W^{+}(Y, c)=S W(Y, c)+k .
$$


The universal term in $\underline{S W}$ is then given by

$$
\left|\operatorname{Tor}\left(H_{1}(Y, \mathbb{Z})\right)\right| \sum_{k=1}^{\infty} k t^{k}=\frac{t}{(1-t)^{2}} \cdot\left|\operatorname{Tor}\left(H_{1}(Y, \mathbb{Z})\right)\right|
$$

where we have included the factor $\left|\operatorname{Tor}\left(H^{2}(Y, \mathbb{Z})\right)\right|=\left|\operatorname{Tor}\left(H_{1}(Y, \mathbb{Z})\right)\right|$ accounting for the different $\operatorname{Spin}^{c}$-structures with the same $\bar{c}_{1}(c)$. A similar computation shows that one obtains the same universal contribution for the Seiberg-Witten invariants computed in the other chamber (i.e. although the individual SW invariants differ, the formal series is the same). We then find,

$$
\underline{S W^{ \pm}}=\frac{t}{(1-t)^{2}} \cdot\left|\operatorname{Tor}\left(H_{1}(Y, \mathbb{Z})\right)\right|+\sum_{x \in H}\left(\sum_{c \mid \bar{c}_{1}(c)=x} S W(Y, c)\right) t^{k}
$$

where the second term in the right hand side is a polynomial, since the SW invariants appearing there are computed for a small perturbation.

The result of Meng and Taubes when $b_{1}(Y)=1$ is that

$$
\underline{S W}^{ \pm}=\tau(Y ; t)
$$

Comparing (2.7) with (3.17) we see that the first term in the Reidemeister-Milnor torsion is the sum of the infinite series of wall-crossing terms. According to (3.18) the remaining piece of the torsion in (2.7), which is a polynomial, must be identified with the SW invariants computed for a small perturbation. Equating the coefficients of the two polynomials in (3.18) and (3.17), one finds:

$$
\sum_{c \mid \bar{c}_{1}(c)=2 k \alpha_{Y}} S W(Y, c)=\sum_{j=1}^{r-|k|} j a_{j+|k|}
$$

in accordance with [17].

\section{Donaldson-Witten partition function for $b_{1}(Y)>1$}

In the next two sections we combine the facts reviewed above with the formulae for the Donaldson-Witten partition function on four-manifolds of the type $X=Y \times \mathbf{S}^{1}$. The extension of the Donaldson-Witten function to non-simply connected four-manifolds was 
begun in [4] [22]. It was completed in [5]. Somewhat later the same result was stated, less precisely, in [23].

The Donaldson-Witten partition function on a four-manifold $X$ depends crucially on $b_{2}^{+}(X)$. As we have noted, the four-manifolds $X=Y \times \mathbf{S}^{1}$ have

$$
b_{2}^{+}(X)=b_{1}(Y)
$$

and hence $Z_{D W}(X)$ depends crucially on $b_{1}(Y)$. For $b_{1}(Y)>1$, the only contribution to the Donaldson-Witten function comes from the SW contributions. It was explicitly verified in [5] that Witten's formula for the Donaldson invariants of simple type manifolds [3] is modified in the nonsimply connected case only when one and three-observables are included. The result for the partition function is then,

$$
Z_{D W}\left(Y \times \mathbf{S}^{1}\right)=2\left(1+i^{-w_{2}^{2}(E)}\right) \sum_{\lambda} e^{2 i \pi\left(\lambda_{0} \cdot \lambda+\lambda_{0}^{2}\right)} S W(\lambda)
$$

Here the sum on $\lambda$ is a sum over $\operatorname{Spin}^{c}$ structures with $c_{1}(c)=2 \lambda, w_{2}(E)$ is an integer lift of the Stiefel-Whitney class of the bundle, and $2 \lambda_{0}=w_{2}(E)$. For $w_{2}(E)=0$, one has to evaluate the sum over all the $\operatorname{Spin}^{c}$ structures, and using (3.4) (3.5) and (2.3) this is just $\Delta_{Y}(1, \ldots, 1)$. We then obtain,

$$
Z_{D W}\left(Y \times \mathbf{S}^{1}\right)=4 \Delta_{Y}(1, \ldots, 1)
$$

which is simply the sum of all the coefficients of the Reidemeister-Milnor torsion.

\subsection{Relation to the Casson-Walker-Lescop invariant}

We will now relate this result to Lescop's extension of the Casson invariant [10], which we will refer to as the Casson-Walker-Lescop invariant $\lambda_{C W L}(Y)$. First, we will recall the surgery operation on a link $\ell$ embedded in a rational homology sphere $R$ to obtain a new three-manifold. To perform surgery on a link, we need to associate to any knot $K_{i}$ in the link $\ell$ a satellite $\mu_{i}$, i.e. a closed curve on the boundary $\partial\left(T\left(K_{i}\right)\right)$ of a tubular neighborhood $T\left(K_{i}\right)$ of $K_{i}$. The homology class of the satellite in the boundary is specified by two rational numbers $\left(p_{i}, q_{i}\right)$ that determine the framing of the knot. $p_{i}$ is the linking number $\operatorname{lk}\left(\mu_{i}, K_{i}\right)$ (i.e. the number of "twists" between the knot and its satellite). If we consider the homology class of the satellite in the solid torus, we have 
that $\left[\mu_{i}\right]=q_{i}\left[K_{i}\right]$. 6 When $R=\mathbf{S}^{3}$, the $\left(p_{i}, q_{i}\right)$ are simply the number of times that the satellite wraps the meridian and parallel, respectively, of the knot $K_{i}$. The quotient $p_{i} / q_{i}$ is the surgery coefficient of the component $K_{i}$. To do the surgery on $\ell$, we consider solid tori $\left(D^{2} \times \mathbf{S}^{1}\right)_{i}$ together with the homeomorphism $h_{i}$ that sends the meridian $\left(\mathbf{S}^{1}\right)_{i}$ to the satellite $\mu_{i}$ on $\partial\left(T\left(K_{i}\right)\right)$. We then cut the solid tori $T\left(K_{i}\right)$ around each of the knots and glue the $\left(D^{2} \times \mathbf{S}^{1}\right)_{i}$ through the homeomorphisms $h_{i}$. In this way we obtain a new three manifold obtained from $R$ through the above surgery presentation. The linking matrix of the presentation is the matrix $L_{i j}$ with components $\operatorname{lk}\left(K_{i}, K_{j}\right)$ when $i \neq j$, and $p_{i} / q_{i}$ when $i=j$.

In order to relate (4.3) to Lescop's extension of the Casson invariant, the key fact is that any oriented, closed three-manifold $Y$ can be obtained by surgery on a link in a rational homology sphere (see [10], Lemma 5.1.1). This link has $b_{1}(Y)$ components and its linking matrix can be chosen to be null (i.e., all its entries are zero). We also have that $\left|H_{1}(R, \mathbb{Z})\right|=\left|\operatorname{Tor}\left(H_{1}(Y, \mathbb{Z})\right)\right|$.

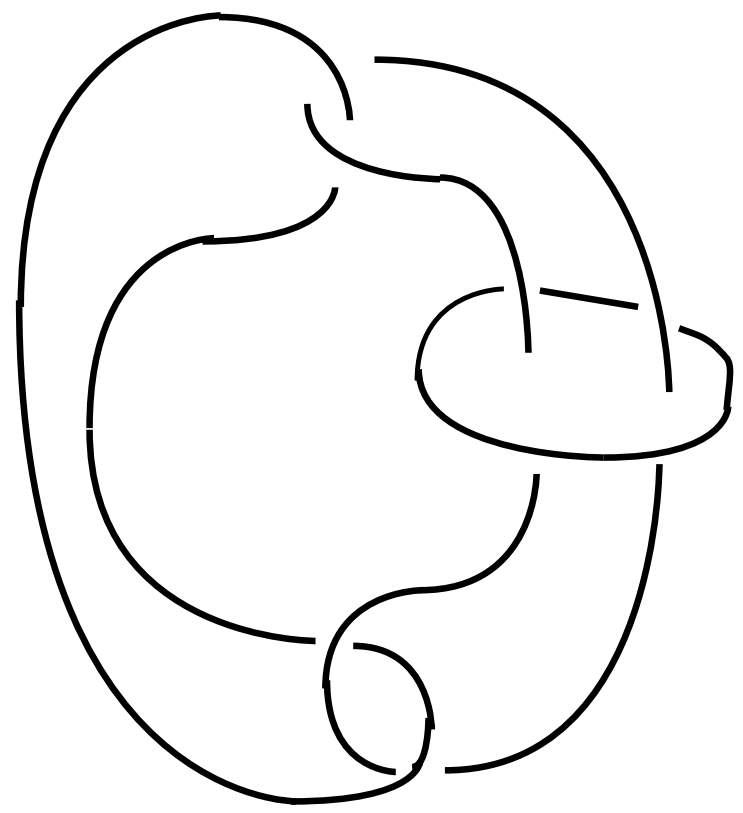

Fig. 3: One presentation of the Borromean link. Note that no two components are linked.

${ }^{6}$ The $q_{i}$ are always integers. If $R=S^{3}$ then the $p_{i}$ are also integers. In general the $p_{i}$ can be rational. 
As an example of this, consider the Borromean link in $\mathbf{S}^{3}$, which has thee components. The linking numbers of its components are zero. Performing surgery on this link, with $p_{i} / q_{i}=0$, we obtain the three torus $\mathbf{T}^{3}$ !

The links considered in these surgery presentations, have the property that the linking number of any two components is zero. Such links are called homology unlinks. For a homology unlink the coefficient $a_{0}$ in (2.10) vanishes (see [10], Remark 5.2.8). Therefore, for homology unlinks the Conway polynomial has the structure:

$$
\nabla_{\ell}(z)=z^{b_{1}+1}\left(a_{1}+a_{2} z^{2}+\ldots+a_{d} z^{2(d-1)}\right)
$$

where $z=t^{1 / 2}-t^{-1 / 2}$ and $b_{1}$ is the number of components of the link. On the other hand, the torsion of $Y$ can be easily computed in terms of the multivariable Conway function of the link, when the linking matrix of the surgery presentation is null. The relation between them is (see [9], section 4.3.4, Remark 2):

$$
\tau\left(Y ; t_{1}, \ldots, t_{b_{1}}\right)=\left(\prod_{i=1}^{b_{1}}\left(t_{i}^{1 / 2}-t_{i}^{-1 / 2}\right)\right)^{-1} \nabla_{\ell}\left(t_{1}, \ldots, t_{b_{1}}\right)
$$

As a consequence of (4.5), notice that, if the manifold $Y$ is obtained by 0 -surgery on a knot $K \subset \mathbf{S}^{3}$, the Alexander polynomial of the manifold $Y$ is the Alexander polynomial of the knot $K$. As an example of (4.5) for links, consider the Borromean link in fig. 3. This link has the multivariable Conway polynomial $\nabla_{l}\left(t_{1}, t_{2}, t_{3}\right)=\prod_{i=1}^{3}\left(t_{i}^{1 / 2}-t_{i}^{-1 / 2}\right)$, therefore the torsion of $\mathbf{T}^{3}$ is $\tau\left(\mathbf{T}^{3}\right)=1$.

Returning to the general case, and using now (2.9), (2.8), (4.4) and (4.5), we obtain for $b_{1}(Y)>1$

$$
\Delta_{Y}(1, \ldots, 1)=\left|H_{1}(R, \mathbb{Z})\right| a_{1}
$$

The right hand side of this expression is precisely Lescop's extension of the Casson invariant for manifolds with $b_{1}(Y)>1$ (see [10], 5.1.7), therefore we have

$$
Z_{D W}\left(Y \times \mathbf{S}^{1}\right)=4 \lambda_{C W L}(Y)
$$

The factor of four arises as follows. The monopole and dyon cusps contribute equally. The other factor of 2 comes from the center of the gauge group $S U(2)$. 


\subsection{Relation to Floer homology}

As we mentioned in the introduction, one of our motivations to analyze the partition function of Donaldson-Witten theory for manifolds with the structure $Y \times \mathbf{S}^{1}$ was to obtain a relation with the Euler characteristic of the Floer cohomology of $Y$. One can check these expectations by considering the three-manifolds $Y_{g}=\Sigma_{g} \times \mathbf{S}^{1}$, where $\Sigma_{g}$ is a Riemann surface of genus $g$. The first Betti number for this class of manifolds is $b_{1}\left(Y_{g}\right)=1+2 g$. The Floer cohomology of these manifolds is computed by turning on a non zero flux on $\Sigma_{g}$, i.e. $w_{2}(E)=\left[\mathbf{S}^{1}\right]$. In this case, the expressions (4.3) (4.7) remain valid, as [14 the basic classes on $Y_{g}$ are two-dimensional classes on $\Sigma_{g}$ and they have no intersection with $w_{2}(E)$. The ring structure of the Floer cohomology for these manifolds is known [24] and in particular $\chi\left(H F\left(Y_{g}\right)\right)=0$ except for $g=1$, where one has $\left.\chi\left(H F\left(\mathbf{S}^{1}\right)^{3}\right)\right)=1$. This is in perfect agreement with the behavior of the Casson invariant, which vanishes for $b_{1}(Y)>3$, and has $\lambda_{C W L}\left(\left(\mathbf{S}^{1}\right)^{3}\right)=1$ [10]. We then see that the partition function should be related to the Euler characteristic of the Floer cohomology, for manifolds with $b_{1}(Y)>1$, as

$$
Z_{D W}\left(Y \times \mathbf{S}^{1}\right)=4 \chi(H F(Y)) .
$$

\subsection{Extension to higher rank}

When $b_{1}(Y)>1$, the partition function of Donaldson-Witten theory for gauge group $S U(N)$ can be easily computed using the results of [6]. In this paper, a simple expression for the $S U(N)$ Donaldson-Witten function on manifolds with $b_{2}^{+}>1$ and of simple type was derived using the $u$-plane approach of $[4$. This expression is given in equation (9.17) of [6]. For the partition function, one obtains the following equation, which generalizes (4.2):

$$
\begin{aligned}
Z_{D W}^{S U(N)}(X)= & N \widetilde{\alpha}_{N}^{\chi} \widetilde{\beta}_{N}^{\sigma} \sum_{k=0}^{N-1} \omega^{k\left[\left(N^{2}-1\right) \delta+N \vec{\lambda}_{0} \cdot \vec{\lambda}_{0}\right]} \\
& \cdot \sum_{\lambda^{I}} \mathrm{e}^{2 \pi i\left(\lambda^{I}, \lambda_{0}^{I}\right)}\left(\prod_{I=1}^{N-1} S W\left(\lambda^{I}\right)\right) \prod_{1 \leq I<J \leq r} q_{I J}^{-\left(\lambda^{I}, \lambda^{J}\right)}
\end{aligned}
$$

In this equation, $\widetilde{\alpha}_{N}, \widetilde{\beta}_{N}$ are constants, $\omega=\exp [i \pi / N], \vec{\lambda}_{0}$ is an integral lifting of the generalized Stiefel-Whitney class of the gauge bundle (see [6] for details), $r=N-1$ is the rank of the gauge group, and $\delta=(\chi+\sigma) / 4$. The terms $q_{I J}$ are the leading terms of the off-diagonal couplings. We have also included an overall $N$ factor corresponding to the order of the center of the gauge group. Finally, the sum over $k$ is a sum over the $\mathcal{N}=1$ vacua. 
If we consider a manifold $X=Y \times \mathbf{S}^{1}$, with $b_{1}(Y)>1$, and we choose $\vec{\lambda}_{0}=0$, the above expression factorizes completely, as the exponents of the nondiagonal couplings $q_{I J}$ are zero. We then find

$$
Z_{D W}^{S U(N)}\left(Y \times \mathbf{S}^{1}\right)=N^{2}\left(\lambda_{C W L}(Y)\right)^{N-1}
$$

which generalizes (4.7) to $S U(N)$. It would be very interesting to compare (4.10) with the generalizations of the Casson invariant that can be obtained using Rozansky-Witten invariants. $\mathrm{O}$ These generalizations might be more nontrivial for the case $b_{1}(Y) \leq 1$.

\section{Three-manifolds with $b_{1}(Y)=1$}

\subsection{The Donaldson-Witten partition function}

When the three-manifold $Y$ has $b_{1}(Y)=1$, the four-manifold $X=Y \times \mathbf{S}^{1}$ has $b_{2}^{+}(X)=$ 1. This means that we have to take into account both the SW and the $u$-plane contribution, as explained in [4]. The $u$-plane contribution for nonsimply connected manifolds has been analyzed in detail in [5]. We also have to take into account that the Donaldson-Witten function depends now on the period point of the metric, and in general our answers will be metric-dependent. We will consider in particular the chambers corresponding to a small or big radius for the circle $\mathbf{S}^{1}$.

When studying the relation between Donaldson invariants and three-dimensional invariants, it is important to take into account the torsion in $H^{2}(X, \mathbb{Z})$. The inclusion of torsion in the $u$-plane integral can be done in the following way: the partition function of the photon includes a sum over topological sectors, i.e. over topological classes of line bundles. This means that we have to sum over torsion classes as well in $H^{2}(X, \mathbb{Z})$. But the photon partition function depends on the topology of the gauge (line) bundle only through the curvature 2-form $F_{A}$ and therefore is only sensitive to the torsion-free part of $H^{2}(X, \mathbb{Z})$. This means that, when summing over all the topological sectors, we will have a sum over the classes in $H^{2}(X, \mathbb{Z}) / \operatorname{Tor}\left(H^{2}(X, \mathbb{Z})\right)$ and then include a global factor $\left|\operatorname{Tor}\left(H^{2}(X, \mathbb{Z})\right)\right|$ multiplying the $u$-plane integral. $\mathrm{O}$ In particular, the wall-crossing formula will have this factor in the presence of torsion, as noticed in [26]. When matching to

7 Investigating generalizations of the Casson invariant for other gauge groups using RozanskyWitten theory has also been recently proposed by G. Thompson in [25].

8 One could define other topological theories by introducing a nontrivial character of the group $\operatorname{Tor}\left(H^{2}(X, \mathbb{Z})\right)$ into the path integral. This would be an analog for Donaldson-Witten theory of "discrete torsion" of a string theory orbifold. We will not investigate this possibility further here. 
SW wall-crossing as in [4] [5], this factor will be present as well on the SW side, as the WC crossing of the SW invariants only depends on the torsion-free part of the $\mathrm{Spin}^{c}$ structure $^{2}$ [20] 21].

We can now compute the $u$-plane contribution to the Donaldson-Witten function of manifolds $X=Y \times \mathbf{S}^{1}$. Let's first analyze the metric dependence. A generic period point has the structure,

$$
\omega=\frac{1}{\sqrt{2}}\left(\mathrm{e}^{\theta}\left[S_{1}\right]+\mathrm{e}^{-\theta}\left[S_{2}\right]\right) .
$$

The limit of a small radius for $\mathbf{S}^{1}$ corresponds to $\theta \rightarrow \infty$, as the volume of the $S_{1}$ is

$$
\int_{S_{1}} \omega=\frac{1}{\sqrt{2}} \mathrm{e}^{-\theta}
$$

The other limit, $\theta \rightarrow-\infty$, corresponds to a large radius for the circle. It is helpful to keep the following example in mind. Suppose $Y$ is a circle bundle over $\mathbb{C} P^{1}$, and $\alpha_{Y}$ is the volume form of a metric $d s^{2}$ on $\mathbb{C} P^{1}$ normalized to unit volume. Then we could consider a metric on $X$ given by:

$$
R^{2}(d \varphi)^{2}+(d \psi)^{2}+\frac{1}{R^{2}} d s^{2}
$$

where $\psi$ is a coordinate on the fiber. Thus we can identify $R$ the radius of the circle parametrized by $0 \leq \varphi \leq 1$ with (5.2).

We first analyze the Donaldson-Witten partition function in the limit $R \rightarrow 0$, and with no magnetic fluxes, so we put $w_{2}(E)=0$. In such a situation, the $u$-plane integral can be computed directly, as in section 8 of $[4]$. For $R \rightarrow 0$, the right choice of the reduction vector $z$ is in this case

$$
z=\left[S_{1}\right], \quad z_{+}^{2}=\frac{1}{2} \mathrm{e}^{-2 \theta} \ll 1 .
$$

Again, if $Y=\mathbf{S}^{2} \times \mathbf{S}^{1}, X=\mathbb{C} P^{1} \times \mathbf{T}^{2}$, this is the chamber where the volume of the torus is very small. As our manifold is non-simply connected, we have to use the expressions of [5]. These involve a choice of cohomology class $\Sigma$ and a modified two-observable $I(\tilde{S})$. In our case the cohomology class $\Sigma$ is given in (3.14) and the modified two-observable is obtained from

$$
(\tilde{S}, z)=(S, z)-\frac{\sqrt{2}}{16} \frac{d \tau}{d u} \Omega
$$

where $\Omega$ is the volume element of the torus $\mathbf{T}^{2}=H^{1}(X, \mathbb{R}) / H^{1}(X, \mathbb{Z})[5]$. The holomorphic function $f$ introduced in 4 [5] is

$$
f=\frac{\sqrt{2}}{8 \pi i} \frac{d u}{d \tau} \exp \left[\frac{\sqrt{2}}{32} a \frac{d \tau}{d a}(S, \Sigma) \Omega+S^{2} T(u)\right]
$$


where $d u / d \tau, a$ and $T(u)$ are certain modular forms described in [4] [5].The $u$-plane integral in this chamber is given by:

$$
Z_{u}=-4 \sqrt{2} \pi i \cdot 2^{9 b_{1} / 4} i^{b_{1} / 2}\left|\operatorname{Tor}\left(H_{1}(Y, \mathbb{Z})\right)\right|\left\{\sum_{I}\left[\frac{f_{I} h_{I}}{1-\mathrm{e}^{-i(\tilde{S}, z) / h_{I}}}\right]_{q^{0}}\right\},
$$

where $f_{I}, h_{I}$ are some more modular forms defined in [4] [5]. The sum is over four regions at infinity of the $u$-plane, labelled $I=(\infty, 0),(\infty, 1),(\infty, 2),(\infty, 3)$, and the monopole and dyon regions of the $u$-plane, labelled $I=M, D$. These regions are each a copy of a fundamental domain for $\mathrm{SL}(2, \mathbb{Z})$. Together the six regions form a fundamental domain for $\Gamma^{0}(4)$. This domain has three cusps: the cusp at infinity (corresponding to the four regions $I=(\infty, 0), \ldots,(\infty, 3))$ and the regions near $\tau=0$ and $\tau=2$ (corresponding to $I=M, D$, respectively.) The numerical prefactor involving $b_{1}$ in (5.7) comes from the measure for the one-forms and was determined in [5] by comparing to known topological results. Using the Künneth theorem and the universal coefficient theorem we have $\operatorname{Tor}\left(H^{2}(X, \mathbb{Z})\right) \cong$ $\operatorname{Tor}\left(H_{1}(Y, \mathbb{Z})\right)$ so the prefactor for the torsion classes can be written as $\left|\operatorname{Tor}\left(H^{2}(X, \mathbb{Z})\right)\right|=$ $\left|\operatorname{Tor}\left(H_{1}(Y, \mathbb{Z})\right)\right|$. As $f$ in $(5.6)$ involves the volume element $\Omega$, we have to expand the functions appearing in (5.7) in $\Omega$ and then integrate. The computation is easy, and one finds that each region $I$ contributes $-2\left|\operatorname{Tor}\left(H_{1}(Y, \mathbb{Z})\right)\right| / 12$. In conclusion, we find

$$
Z_{u}\left(Y \times \mathbf{S}^{1}\right)=-\left|\operatorname{Tor} H_{1}(Y, \mathbb{Z})\right|
$$

Let's now consider the SW contribution. The SW invariants, computed for a small perturbation, do not depend on the metric. To obtain the Donaldson-Witten partition function, we have to sum over all SW invariants, as in (4.9). Using (2.5) and (3.19), we find

$$
\sum_{c} S W(Y, c)=\sum_{\ell=1}^{r} \ell^{2} a_{\ell}=\frac{1}{2} \Delta_{Y}^{\prime \prime}(1),
$$

where $\Delta_{Y}$ is the Alexander polynomial. Taking into account (2.6), we can write the partition function of Donaldson-Witten theory, $Z_{D W}=Z_{u}+Z_{S W}$, in terms of the Alexander polynomial of $Y$ as follows:

$$
Z_{D W}\left(Y \times \mathbf{S}^{1}\right)=2 \Delta_{Y}^{\prime \prime}(1)-\Delta_{Y}(1)
$$

It is interesting to compare this result with the Casson invariant as extended by Lescop [10]. For manifolds with $b_{1}(Y)=1$ it is given by (see [10], 5.1):

$$
\lambda_{C W L}(Y)=\frac{1}{2} \Delta_{Y}^{\prime \prime}(1)-\frac{\left|\operatorname{Tor}\left(H_{1}(Y, \mathbb{Z})\right)\right|}{12} .
$$


We therefore arrive at one of the key results of this paper:

$$
Z_{D W}\left(Y \times \mathbf{S}^{1}\right)=4 \lambda_{C W L}(Y)-\frac{4}{6}\left|\operatorname{Tor}\left(H_{1}(Y, \mathbb{Z})\right)\right| .
$$

Note that, even after accounting for a factor of 4, as in (4.7), the invariants do not agree. It is important to notice that the result (5.10) is obviously an integer, while Lescop's extension of the Casson invariant for manifolds with $b_{1}(Y)=1$ takes values in $\mathbb{Z} / 12$. For instance, for $Y=\mathbf{S}^{2} \times \mathbf{S}^{1}$ (which has $\Delta_{Y}(t)=1$ ), one has $\lambda_{C W L}(Y)=-1 / 12$, but $Z_{D W}\left(Y \times \mathbf{S}^{1}\right)=-1$. We will comment on this disagreement below, as well as on the relation of (5.10) to the results of [8].

The fact that our result is an integer suggests that it is related to the Euler characteristic of the Floer homology of $Y$. Strictly speaking, we should expect to recover the Euler characteristic of the Hilbert space in the chamber $R \rightarrow \infty$ (the "long neck" chamber). However, one can easily check that, in this chamber, one also has (5.10) for the partition function. This is easily seen by using the wall-crossing formulae derived in [5] for the Donaldson invariants: there is no wall-crossing for the partition function. This interpretation of (5.10) as an Euler characteristic is not easy to check from known mathematical results, however, as on manifolds with $b_{1}(Y)>0$ the Floer homology has only been constructed when there is a nontrivial magnetic flux on $Y$, in order to avoid reducible flat connections 27] (see [28] for a nice review). In order to interpret our result (5.10), it is illuminating to compute the partition function when $w_{2}(E)$ has the integral lift $\alpha_{Y}$. We can do the computation in two different ways. When one uses the lattice reduction and unfolding, the inclusion of the flux $w_{2}(E)=\alpha_{Y}$ has the following effect: the contribution of the monopole and the dyon cusps is the same as before, but for the cusps at infinity, one has to change

$$
\frac{1}{1-\mathrm{e}^{-i(\tilde{S}, z) / h_{I}}} \rightarrow \frac{1}{2 i} \csc \left(\frac{(\tilde{S}, z)}{2 h_{I}}\right) .
$$

After doing this, the contribution from the monopole and the dyon cancel the contribution from the four semiclassical regions. Therefore, the $u$-plane integral vanishes. Alternatively, one can consider the chamber $R \rightarrow \infty$, where the vanishing theorem for the $u$-plane integral holds. As there is no wall-crossing for the partition function, we find again $Z_{u}=0$. Therefore,

$$
Z_{D W}^{w_{2}=\alpha_{Y}}\left(Y \times \mathbf{S}^{1}\right)=2 \Delta_{Y}^{\prime \prime}(1)
$$

We see that the inclusion of a nonzero flux, which gets rid of the reducibles, kills the $u$-plane contribution, as expected. The term $-\Delta_{Y}(1)$ should be understood as the contribution of the reducible flat connections on $Y$ to the partition function. 


\subsection{A relation to Reidemeister-Milnor torsion}

In the above computations, we have not included any observable in the generating function. One can try to include an appropriate 2-observable in such a way that the Donaldson-Witten function has the structure of a formal series related to the Meng-Taubes series. When a 2-observable is included, the SW contribution for $Y \times \mathbf{S}^{1}$ has the structure [3] [5]

$$
Z_{S W}\left(\mathrm{e}^{I(S)}\right)=2 \sum_{\lambda} S W(\lambda)\left(\mathrm{e}^{(S, x)+S^{2} / 2}+\mathrm{e}^{-i(S, x)-S^{2} / 2}\right),
$$

where we have put $w_{2}(E)=0$, the sum is over $\operatorname{Spin}^{c}$ structures and $x=2 k \alpha_{Y}$. If we consider the 2-homology class

$$
S=\frac{1}{2} t S_{2}
$$

where $t$ has to be understood as a formal parameter, we see that the dependence in $t$ has the form

$$
\mathrm{e}^{(S, x)}=\left(\mathrm{e}^{t}\right)^{k},
$$

for the monopole contribution. Therefore, the sum of SW invariants corresponding to the $\operatorname{Spin}^{c}$ structures with $x=2 k \alpha_{Y}$ are the coefficients of a polynomial in $\mathrm{e}^{t}$ (for the monopole contribution) and in $\mathrm{e}^{-i t}$ (for the dyon contribution), very much as in (3.12), and (5.15) becomes

$$
\sum_{x \in H}\left(\sum_{c \mid \bar{c}_{1}(c)=x} S W(Y, c)\right)\left(\left(\mathrm{e}^{t}\right)^{k}+\left(\mathrm{e}^{-i t}\right)^{k}\right) .
$$

Notice that the SW invariants considered here are computed using a small perturbation. The surprise comes when one computes the $u$-plane contribution. We have to expand

$$
\frac{1}{1-\mathrm{e}^{-i(\tilde{S}, z) / h}}=\frac{1}{1-\mathrm{e}^{-i(S, z) / h}}+\frac{\mathrm{e}^{-i(S, z) / h}}{\left(1-\mathrm{e}^{-i(S, z) / h}\right)^{2}} \frac{i \sqrt{2}}{16} \frac{d \tau}{d a} \Omega+\ldots,
$$

and only the second term survives after integrating over the 2-torus of flat connections. We have to extract the $q^{0}$ power of the expansions at the different cusps. The monopole and dyon cusp contributions are regular at $q=0$, while the semiclassical cusp gives a power series in $h_{\infty}(q)$, where $h_{\infty}$ is a modular form given in [4]. The final result is

$$
\begin{aligned}
Z_{D W}\left(\mathrm{e}^{I(S)}\right) & =2 \frac{\left|\operatorname{Tor}\left(H_{1}(Y, \mathbb{Z})\right)\right|}{\left(\left(\mathrm{e}^{t}\right)^{1 / 2}-\left(\mathrm{e}^{t}\right)^{-1 / 2}\right)^{2}}+2 \sum_{x \in H}\left(\sum_{c \mid \bar{c}_{1}(c)=x} S W(Y, c)\right)\left(\mathrm{e}^{t}\right)^{k} \\
& +2 \frac{\left|\operatorname{Tor}\left(H_{1}(Y, \mathbb{Z})\right)\right|}{\left(\left(\mathrm{e}^{-i t}\right)^{1 / 2}-\left(\mathrm{e}^{-i t}\right)^{-1 / 2}\right)^{2}}+2 \sum_{x \in H}\left(\sum_{c \mid \bar{c}_{1}(c)=x} S W(Y, c)\right)\left(\mathrm{e}^{-i t}\right)^{k} \\
& -\left[\frac{2\left|\operatorname{Tor}\left(H_{1}(Y, \mathbb{Z})\right)\right|}{\left(\sin \left(t / 4 h_{\infty}\right)\right)^{2}}\right]_{q^{0}} .
\end{aligned}
$$


This expression is regular when $t=0$, as the poles cancel between the monopole and dyon cusps. We can write it in a more compact form using (3.17) and (3.18):

$$
Z_{D W}\left(\mathrm{e}^{I(S)}\right)=2 \tau\left(Y ; \mathrm{e}^{t}\right)+2 \tau\left(Y ; \mathrm{e}^{-i t}\right)-\left[\frac{2\left|\operatorname{Tor}\left(H_{1}(Y, \mathbb{Z})\right)\right|}{\left(\sin \left(t / 4 h_{\infty}\right)\right)^{2}}\right]_{q^{0}} .
$$

We see that the infinite series associated to the stable SW invariants can be reinterpreted as the $u$-plane contribution from the monopole or dyon cusps (in the chamber $R \rightarrow 0$ ) to the generating function associated to the observable (5.16). In addition, we have found a relation between the Reidemeister-Milnor torsion and a generating function in DonaldsonWitten theory. It is interesting to notice that Donaldson-Witten functions involving $\mathrm{e}^{t I(S)}$ appear in a natural way in the context of Fukaya-Floer homology (see for instance [28].)

We should point out that the generalization of the results obtained here for $b_{1}(Y)=1$ to the higher rank case is not an easy task, since the computation of the integral over the Coulomb branch can not be done using the unfolding technique.

\section{On the perils of compactification}

We now return to the key result (5.12) and investigate its meaning.

\subsection{Review of the relation of three- and four-dimensional SYM}

In this section we review some results of Seiberg and Witten [7] and of Rozansky and Witten [8].

In [7] Seiberg and Witten studied the low-energy effective action of $\mathcal{N}=2$ super Yang-Mills compactified on $\mathbb{R}^{3} \times \mathbf{S}^{1}$ where the $S^{1}$ factor has radius $R$. They argued that in the limits $R \rightarrow \infty$ and $R \rightarrow 0$ one recovers the pure $4 \mathrm{~d}$ and $3 \mathrm{~d}$ theories, respectively, and therefore that the two different limits are connected through an interpolating theory that depends on $R$. The low-energy description of the compactified theory is a threedimensional $\mathcal{N}=4$ sigma model whose target space is a hyperkähler manifold $\mathcal{M}_{R}$. As a complex manifold $\mathcal{M}_{R}$ can be identified with the total space of the elliptic fibration over the $u$-plane defined by the SW curve. The metric on $\mathcal{M}_{R}$ depends on the compactification radius $R$ and has not been determined explicitly for general values of $R$. In the limit as $R \rightarrow 0$ there is a well-defined limit on the complement of a section of the elliptic fibration and the limiting metric turns out to be the Atiyah-Hitchin metric. 
The derivation of the sigma model with target $\mathcal{M}_{R}$ can be approached in two ways: One can first work out a low energy theory in 4 dimensions and then compactify, or one can compactify and then work out the quantum corrections. The first approach is better at large $R$ and the second is better at small $R$. We elaborate on this briefly.

The first method uses the compactification of the low-energy SW effective theory of a $U(1)$ vectormultiplet [7]. In this point of view we first first work in four dimensions and go to the infrared. To write the low energy lagrangian we choose a duality frame, i.e., we use $S L(2, \mathbb{Z})$ to make a choice of weakly coupled $U(1)$ vectormultiplet $(a(u), A, \lambda)$. We next carry out dimensional reduction. Then we use 3-dimensional dualization to go from the $3 \mathrm{D}$ vector field $A_{\mu}$ to a compact scalar $\sigma$. The result is the tree level sigma model:

$$
\int_{Y} 2 \pi R g_{u \bar{u}} d u \wedge * d \bar{u}+\frac{1}{8 \pi^{2} R \operatorname{Im} \tau(u)}|d \sigma-\tau d b|^{2}
$$

where $0 \leq \sigma, b \leq 2 \pi$. $⿴$ Thus, the sigma model has as target the total space of the elliptic fibration over the $u$-plane. The metric in (6.1) is only an approximation. However, the underlying complex manifold is exactly determined. As a complex manifold the total space over the $u$-plane is the surface

$$
z y^{2}=x^{3}-z x^{2} u+z^{2} x
$$

for $((x: y: z) ; u) \in \mathbb{C} P^{2} \times \mathbb{C}$. As shown in [7], after removing a section of the fibration one may identify this surface with the Atiyah-Hitchin manifold in one of its complex structures. Unfortunately, there are important quantum corrections and Kaluza-Klein corrections which are hard to control in this approach.

Working instead at small $R$ one can make a compactification of the the underlying $\mathrm{UV} S U(2) \mathcal{N}=2, d=4$ theory, and then work out the quantum dynamics. In the limit $R \rightarrow 0$ we expect that we can use the dimensional reduction of the UV theory to obtain $S U(2) \mathcal{N}=4, d=3 \mathrm{SYM}$. In this theory one can study quantum corrections. Denoting the scalar field vevs in the Cartan subalgebra by $\vec{\phi}$, and working at large $|\vec{\phi}|$ and to one-loop approximation one finds a Taub-Nut metric [29][7]:

$$
d s^{2}=V^{-1}(d \sigma+\vec{\omega} \cdot d \vec{\phi})^{2}+V(d \vec{\phi})^{2}
$$

9 There is one important difference relative to [7]. In our case the threefold $Y$ is compact, with a volume growing like $\operatorname{vol}(Y) \sim R^{-1}$. 
for the target space of the 3D sigma model. Here $\sigma$ is the dualized photon and, as usual, $\nabla \times \vec{\omega}=\nabla V[30$. Moreover in this case the TN potential here has negative mass:

$$
V=\frac{2 \pi}{e_{3}^{2}}-\frac{1}{2 \pi|\vec{\phi}|}
$$

Furthermore, studies of 3D instanton effects reveal the leading $e^{-r}$ corrections corresponding to the Atiyah-Hitchin metric [31].

Motivated by the non-perturbative results in supersymmetric gauge theory in three dimensions of [7], Rozansky and Witten constructed a new topological field theory in three dimensions [8]. 10 The RW theory is based on the twisting of an $\mathcal{N}=4$ sigma model with target space a hyperkähler manifold $\mathcal{M}$. It has been known for some time that the partition function of the twisted $\mathcal{N}=4$ Yang-Mills theory in three dimensions (which is the dimensional reduction of Donaldson-Witten theory) formally computes the Casson invariant, [32] [33] 34] [35]. In [8], Rozansky and Witten used the low-energy description of this theory to show that this is not just formally true, but really true in the case of homology spheres, while for three manifolds with $b_{1}(Y)>0$, the Rozansky-Witten partition function is precisely Lescop's extension of the Casson invariant:

$$
Z_{R W}\left(Y ; \mathcal{M}_{0}\right)=\lambda_{C W L}(Y)
$$

More generally, we may use the interpolating hyperkähler manifold $\mathcal{M}_{R}$ of the SW 3D sigma model to obtain:

$$
Z_{R W}\left(Y ; \mathcal{M}_{R}\right)=-\frac{1}{2} b_{\theta}\left(\mathcal{M}_{R}\right) \lambda_{C W L}(Y)
$$

where [8]

$$
b_{\theta}\left(\mathcal{M}_{R}\right)=\int_{\mathcal{M}_{R}} \frac{1}{8 \pi^{2}} \operatorname{Tr}[\mathcal{R} \wedge \mathcal{R}],
$$

and $\mathcal{R}$ is the curvature two-form associated to the hyperkähler metric on $\mathcal{M}_{R}$. For $R=0$, $\mathcal{M}_{R}$ is the Atiyah-Hitchin manifold, and the integral is -2 .

10 A new paper on the subject, with some relation to the present paper, was recently posted on the e-print archives [25]. 


\subsection{Donaldson-Witten $\neq$ Rozansky-Witten}

We have considered the partition function of Donaldson-Witten theory on the fourmanifold $X=Y \times \mathbf{S}^{1}$ in the limit when the radius of the circle goes to zero. For manifolds with $b_{1}(Y)=1$ our result does not agree with Lescop's extension of the Casson invariant, and therefore does not agree with the Rozansky-Witten partition function. However, the results are not totally unrelated and we can be more precise about the relation between the different quantities.

In general, the Donaldson-Witten function has the structure

$$
Z_{D W}=Z_{u}+Z_{S W}
$$

but there is no canonical decomposition into a " $u$-plane part" and an "SW part." As we change the metric the relative contributions change due to SW wall-crossing. However, when there is no SW wall-crossing, as in the present case, the decomposition of the Donaldson-Witten function in terms of SW contributions and the $u$-plane integral is canonical, since they do not mix.

Moreover, when we perform the computation of $Z_{u}$ in a chamber such as $R \rightarrow 0$ by lattice reduction and unfolding, the contributions from the different regions on the $u$-plane are well distinguished: the contribution of monopole and dyon cusps correspond to finite regions in the $u$-plane centered around the monopole and dyon singularities, respectively, while the four semiclassical cusps correspond to regions that extend to infinity in the $u$-plane. Thus, given a chamber, we have the decomposition

$$
Z_{u}=Z_{u, M}+Z_{u, D}+Z_{u, \infty} .
$$

It is important to stress that, in general, the decomposition of the $u$-plane integral into contributions from different cusps is not canonical and depends on the chamber under consideration (when the integral is computed using lattice reduction, this decomposition depends on the chamber through the choice of a lattice vector $z$, as explained in section 8 of 四). However, in the present case, for both $R \rightarrow 0$ and $R \rightarrow \infty$ we find

$$
Z_{u}=-\left|\operatorname{Tor}\left(H_{1}(Y, \mathbb{Z})\right)\right|
$$


because there is neither Donaldson nor SW wall-crossing. More surprisingly, we find for $R \rightarrow 0, \infty$ the same decomposition: 11

$$
\begin{aligned}
& Z_{u, M}=Z_{u, D}=-\frac{1}{6}\left|\operatorname{Tor}\left(H_{1}(Y, \mathbb{Z})\right)\right| \\
& Z_{u, \infty}=-\frac{4}{6}\left|\operatorname{Tor}\left(H_{1}(Y, \mathbb{Z})\right)\right| .
\end{aligned}
$$

Combining these two decompositions we can write a decomposition of the DonaldsonWitten function for the chamber $R \rightarrow 0$

$$
Z_{D W}=Z_{M}+Z_{D}+Z_{\infty}
$$

For example, the contribution of the monopole cusp is given by:

$$
Z_{M}=2\left(\frac{1}{2} \Delta_{Y}^{\prime \prime}(1)-\frac{\left|\operatorname{Tor}\left(H_{1}(Y, \mathbb{Z})\right)\right|}{12}\right)=2 \lambda_{C W L}(Y)
$$

Here the first term comes from the SW invariants at $u=1$, and the second term comes from the contribution of the monopole cusp in the $u$-plane integral. The same result holds for $Z_{D}$. Therefore, this "truncated" topological invariant agrees with the Rozansky-Witten invariant (after including in the latter the factor of 2 due to the center of the gauge group), and therefore with Lescop's extension of the Casson invariant. In comparing the theories we therefore have:

$$
\begin{aligned}
Z_{M}\left(Y \times \mathbf{S}^{1}\right) & =2 Z_{R W}(Y) \\
Z_{D}\left(Y \times \mathbf{S}^{1}\right) & =2 Z_{R W}(Y) \\
Z_{\infty}\left(Y \times \mathbf{S}^{1}\right) & =-\frac{4}{6}\left|\operatorname{Tor}\left(H_{1}(Y, \mathbb{Z})\right)\right| \not<2 Z_{R W}(Y)
\end{aligned}
$$

If we include the two-observable $(5.16)$ and use $(5.20)$ we find that $Z_{M}$ is given by

$$
Z_{M}\left(\mathrm{e}^{I(S)}\right)=2 \tau\left(Y ; \mathrm{e}^{t}\right)
$$

11 There is a very interesting further subtlety here. If we also "regularize" by including a twoobservable $I(S)$ as in $(5.20)$ then we find that $Z_{u}\left(e^{I(S)}\right)$ is different in the chambers $R \rightarrow 0$ and $R \rightarrow$ $\infty$. Still when we subsequently take the limit $S \rightarrow 0$ we obtain the same result: $\lim _{S \rightarrow 0} Z_{u}\left(e^{I(S)}\right)=$ $-\left|\operatorname{Tor}\left(H_{1}(Y, \mathbb{Z})\right)\right|$ in both chambers. Nevertheless, if we first take $R \rightarrow \infty$ and then let $S \rightarrow 0$ we find a different decomposition of the $u$-plane integral: $Z_{u, \infty}=-\left|\operatorname{Tor}\left(H_{1}(Y, \mathbb{Z})\right)\right|, Z_{u, M}=0$, $Z_{u, D}=0$. 
It is interesting to notice that the second term in (6.13) can be interpreted as the $\zeta$ regularization of (6.15) as $t \rightarrow 0$. The infinite series one obtains in this limit is precisely the infinite series of wall-crossings (3.16):

$$
\left|\operatorname{Tor}\left(H_{1}(Y, \mathbb{Z})\right)\right|(1+2+\ldots)=\zeta(-1)\left|\operatorname{Tor}\left(H_{1}(Y, \mathbb{Z})\right)\right|=-\frac{\left|\operatorname{Tor}\left(H_{1}(Y, \mathbb{Z})\right)\right|}{12}
$$

A glance at (5.20) shows that the cusp at infinity does not contribute anything like the torsion. It remains to understand more clearly why there is a discrepancy between three-dimensional and four-dimensional theories. Note that this subtlety does not enter for $b_{1}(Y)>1$. In this case there is no $u$-plane contribution and the $4 \mathrm{D}$ and $3 \mathrm{D}$ theories are related in the expected way. Therefore, we begin by revisiting the $u$-plane integral.

\subsection{A closer look at the u-plane measure}

Let us now examine more closely the $u$-plane integral:

$$
\begin{gathered}
Z_{u}\left(Y \times \mathbf{S}^{1}\right)=\frac{1}{2}\left|\operatorname{Tor}\left(H_{1}(Y, \mathbb{Z})\right)\right| \int_{\Gamma^{0}(4) \backslash \mathcal{H}} \frac{d x d y}{y^{1 / 2}} . \\
\sum_{n, m \in \mathbb{Z}}\left\{\left(\mathrm{e}^{\theta}\left(n^{2} \mathrm{e}^{-2 \theta}-m^{2} \mathrm{e}^{2 \theta}\right)-\mathrm{e}^{\theta} \frac{1}{2 \pi y}\right) \cdot \exp \left[-\pi y\left(n^{2} \mathrm{e}^{-2 \theta}+m^{2} \mathrm{e}^{2 \theta}\right)-2 \pi i m x\right]\right\}
\end{gathered}
$$

The integral is over a fundamental domain for the congruence subgroup $\Gamma^{0}(4)$. We denote $\tau=x+i y$. The sum is over line bundles for the $U(1)$ gauge theory with

$$
\lambda=n\left[S_{1}\right]+m\left[S_{2}\right] \quad n, m \in \mathbb{Z}
$$

Recall that the metric defines the period point $* \omega=\omega$ with

$$
\omega=\frac{1}{\sqrt{2}}\left(\mathrm{e}^{\theta}\left[S_{1}\right]+\mathrm{e}^{-\theta}\left[S_{2}\right]\right)
$$

The first term in the sum in (6.17) comes from bringing down the term $\sim \frac{d \tau}{d a} F \psi \psi$ in the action and soaking up fermion $\psi$-zeromodes associated with $b_{1}(X)=2$. The second term in the sum in (6.17) is a contact term.

Referring to the definitions of the classes $\left[S_{1}\right],\left[S_{2}\right]$ and to $(5.2)(5.3)$ we see that the limit of shrinking Kaluza-Klein circle $R \sim e^{-\theta} \rightarrow 0$ corresponds to $\theta \rightarrow \infty$. Let us now consider the behavior of $Z_{u}$ in this limit.

The first thing to note is that the terms in the integrand with $m=0$ actually blow up in this limit! The reason for this is that such line bundles have "instanton" connections 
with no dependence in the Kaluza-Klein circle direction $\varphi$. However, since the overall volume is fixed, the volume of $Y$ goes like $e^{+\theta} \rightarrow+\infty$. Thus, new zeromodes, related to the decompactification of $Y$ develop, causing a term by term divergence in the integrand of (6.17). Interestingly, there is in fact a cancellation between the positive contribution of the fermion zeromode term and the negative contribution of the contact term. This can be seen mathematically as follows.

First, note that the two terms in the sum in (6.17) combine as a total derivative in $\theta$ :

$$
\frac{e^{2 \theta}}{2 \pi y} \frac{d}{d \theta}\left[e^{-\theta} \sum_{n, m} \exp \left\{-\pi y\left(n^{2} \mathrm{e}^{-2 \theta}+m^{2} \mathrm{e}^{2 \theta}\right)-2 \pi i m x\right\}\right]
$$

Now we see that the divergence from the sum on $n$ (at $m=0$ ) can be offset by the vanishing of $e^{-\theta}$. To see which dominates we use the Poisson summation formula to write:

$$
\sum_{n, m} \exp \left[-\pi y\left(n^{2} \mathrm{e}^{-2 \theta}+m^{2} \mathrm{e}^{2 \theta}\right)-2 \pi i m x\right]=\frac{\mathrm{e}^{\theta}}{y^{1 / 2}} \sum_{\hat{n}, m} \exp \left[-\frac{\pi}{y}|\hat{n}+m \tau|^{2} \mathrm{e}^{2 \theta}\right] .
$$

Combining the two terms one sees that (6.17) becomes

$$
Z_{u}=-\frac{1}{2}\left|\operatorname{Tor}\left(H_{1}(Y, \mathbb{Z})\right)\right| \int_{\Gamma^{0}(4) \backslash \mathcal{H}} \frac{d x d y}{y^{3}} \mathrm{e}^{4 \theta} \sum_{\hat{n}, m}|\hat{n}+m \tau|^{2} \exp \left[-\frac{\pi}{y}|\hat{n}+m \tau|^{2} \mathrm{e}^{2 \theta}\right] .
$$

We can learn two things from (6.22). First, we now note that not only have the divergences of the $m=0$ terms cancelled, but the remaining integrand actually vanishes exponentially fast as $\theta \rightarrow+\infty$ :

$$
\lim _{\theta \rightarrow+\infty} \mathrm{e}^{4 \theta} \sum_{\hat{n}, m}|\hat{n}+m \tau|^{2} \exp \left[-\frac{\pi}{y}|\hat{n}+m \tau|^{2} \mathrm{e}^{2 \theta}\right]=0
$$

The second thing we learn from $(6.22)$ is that the measure is in fact $S L(2, \mathbb{Z})$ invariant. This is a surprise since in general the $u$-plane measure is only $\Gamma^{0}(4)$ invariant. Thus, each of the six copies of the fundamental domain $\mathcal{F}$ of $S L(2, \mathbb{Z})$ contribute equally to $Z_{u}$. Moreover, the integrand is in the standard form for which one can apply the unfolding technique. Combining these two observations we get:

$$
Z_{u}=-3\left|\operatorname{Tor}\left(H_{1}(Y, \mathbb{Z})\right)\right| \int_{0}^{\infty} \frac{d y}{y^{3}} \mathrm{e}^{4 \theta} \sum_{\hat{n}} \hat{n}^{2} \exp \left[-\pi \hat{n}^{2} \frac{e^{2 \theta}}{y}\right]
$$


We can some further insight into the nature of the measure from the expression (6.24). Note first that we can explicitly eliminate all $\theta$-dependence by a change of variables to

$$
\xi \equiv \frac{e^{2 \theta}}{y}=\frac{1}{2 R^{2} y}
$$

Thus the integral (6.24) is in fact $R$-independent and simply given by $-\left|\operatorname{Tor}\left(H_{1}(Y, \mathbb{Z})\right)\right|$. As we have observed, and as is even more obvious from (6.24), at fixed value of $y$, as $R \rightarrow 0$ the integrand vanishes. On the other hand the integral is $R$-independent and nonzero. Thus the integrand is becoming delta function supported. We can see this rather explicitly by letting $w=1 / y$ and noting that:

$$
\begin{aligned}
\lim _{R \rightarrow 0}\left(\sum_{\hat{n} \in \mathbb{Z}} \hat{n}^{2} \frac{w}{R^{4}} e^{-\pi \hat{n}^{2} w / R^{2}}\right) d w & =\sum_{\hat{n} \neq 0} \lim _{R \rightarrow 0} \hat{n}^{2} \frac{w}{R^{4}} e^{-\pi \hat{n}^{2} w / R^{2}} d w \\
& =\sum_{\hat{n} \neq 0} \frac{1}{\pi^{2} \hat{n}^{2}} \delta(w) d w \\
& =\frac{1}{3} \delta(w) d w
\end{aligned}
$$

Thus, as $R \rightarrow 0$, the measure in each cusp region is becoming $\delta$-function supported at $y=\infty .12$ Now, this discussion can be carried out in each of the three cusp regions of the fundamental domain of $\Gamma^{0}(4)$. In each cusp region we have a different $q$ expansion $q=e^{2 \pi i \tau}, \tau=x+i y$. Denoting the relevant $q$-parameters by $q_{M}, q_{D}, q_{\infty}$ the $R \rightarrow 0$ limit of the $u$-plane measure is

$$
-\left|\operatorname{Tor}\left(H_{1}(Y, \mathbb{Z})\right)\right|\left\{\frac{1}{6} \delta^{(2)}\left(q_{M}\right) d^{2} q_{M}+\frac{1}{6} \delta^{(2)}\left(q_{D}\right) d^{2} q_{D}+\frac{4}{6} \delta^{(2)}\left(q_{\infty}\right) d^{2} q_{\infty}\right\} .
$$

\subsection{An interpretation of the result}

Given the facts reviewed in section 6.1, the discrepancy between $Z_{D W}\left(Y \times \mathbf{S}^{1}\right)$ and $Z_{R W}(Y)$ is somewhat surprising. In this section we will discuss some of the physics behind this discrepancy and suggest an interpretation of the result. We thank N. Seiberg and E. Witten for important remarks that helped us to this picture.

Let us first dispose of a red-herring. Nonintegral values of the Witten index are often associated with the presence of noncompact field spaces, and the mishandling of a "bulk"

12 This is similar to the source of the holomorphic anomaly in certain one-loop expressions in string theory [36]. 
or a "boundary" contribution. We stress that this is not what is going on here since $Z_{D W}\left(Y \times \mathbf{S}^{1}\right)$ has no wall crossing.

Our interpretation of (6.14) is that the Donaldson-Witten theory on $X=Y \times \mathbf{S}^{1}$ for small $R$ is simultaneously a three-dimensional and a four-dimensional theory. By this we mean the following: We must integrate over moduli space to get the physical partition function. There is very different physics in the different regimes of moduli space. Some of it is three-dimensional and some of it is four-dimensional.

For small $R$ the measure for the cusp at $\infty$ is concentrated in the region

$$
\operatorname{Im} \tau_{\infty}(u) \gtrsim 1 / R^{2}
$$

where $\tau_{\infty}$ is the $\tau$ parameter selected by the semiclassical cusp. Because of asymptotic freedom, at small $R$ we can use the semiclassical one-loop answer and the measure is concentrated in the region

$$
\log |u| \gtrsim \frac{\pi}{2 R^{2}}
$$

In this region of the $u$-plane physics is effectively four-dimensional. The infrared 4dimensional SW description becomes applicable at length scales

$$
\ell \sim \frac{1}{\sqrt{u}} \sim \exp \left[-\frac{\pi}{4 R^{2}}\right] \ll R
$$

At such length scales the compactification on $\mathbf{S}_{R}^{1}$ is completely irrelevant. Because of asymptotic freedom this becomes better and better as $R \rightarrow 0$.

Let us now consider the monopole cusp. The $u$-plane measure is concentrated in the region

$$
\operatorname{Im} \tau_{M}(u) \gtrsim 1 / R^{2}
$$

where $\tau_{M}=-1 / \tau_{\infty}$ defines the weak-coupling frame near the monopole cusp $u=1$. In particular $\tau_{M}(u) \cong \frac{1}{i \pi} \log (u-1)$ so the relevant region of the $u$-plane is:

$$
|u-1| \lesssim e^{-\pi / R^{2}}
$$

consequently the monopoles are very light.

However, the effective theory of monopole hypermultiplets and dual $U(1)$ vectormultiplets is IR free and UV unstable - it is not defined as a four-dimensional theory at distance scales $\ell \ll R$. Indeed, the infrared SW description is only applicable at length scales

$$
\ell \gtrsim e^{+\pi / R^{2}} \gg R
$$

For this region of moduli space we must first compactify and then solve for the dynamics. 


\subsection{Comments on one-loop corrections}

When one combines standard one-loop expressions with some of the above remarks one can be lead to paradoxes which have troubled the authors of this paper not a little. In this section we mention some of these confusions, and suggest a resolution.

In classical dimensional reduction the gauge couplings $e_{3}^{2}$ and $g_{4}^{2}$ in three and four dimensions, respectively, are related by $g_{4}^{2}=e_{3}^{2} R$.

In 4D gauge theory, when integrating out massive charged vectormultiplets and hypermultiplets of mass $m_{i}$ and charge $Q_{i}$ in a weakly coupled theory the threshold correction

relating the coupling $g_{4, U V}^{2}$ of the underlying theory and $g_{4, I R}^{2}$ of the low energy effective theory is:

$$
\frac{8 \pi}{g_{4, I R}^{2}}=\frac{8 \pi}{g_{4, U V}^{2}}-16 \pi \sum_{i}(-1)^{\epsilon_{i}} Q_{i}^{2} \int \frac{d^{4} p}{(2 \pi)^{4}} \frac{1}{\left(p^{2}+m_{i}^{2}\right)^{2}}
$$

where $\epsilon_{i}=0$ for VM's and $\epsilon_{i}=1$ for HM's. The integral in (6.34) is log divergent and a regularization

$$
\frac{1}{\left(p^{2}+m_{i}^{2}\right)^{2}} \rightarrow \frac{\Lambda^{2 \alpha-4}}{\left(p^{2}+m_{i}^{2}\right)^{\alpha}}
$$

with $\alpha=2+\epsilon, \epsilon \rightarrow 0^{+}$is understood here and below.

When we compactify $\mathbb{R}^{4} \rightarrow \mathbb{R}^{3} \times \mathbf{S}^{1}$ the integral in (6.34) becomes

$$
\frac{1}{R} \sum_{n=-\infty}^{\infty} \int \frac{d^{3} \vec{p}}{(2 \pi)^{3}} \frac{1}{\left(\vec{p}^{2}+\left(A_{4}+n / R\right)^{2}+m^{2}\right)^{2}}
$$

where $A_{4}$ is a background Wilson loop. The expression (6.36) interpolates nicely between the renormalizations in $3 \mathrm{D}$ and $4 \mathrm{D}$. 3 Indeed, performing the integral on $\vec{p}$ we get:

$$
\frac{\pi^{3 / 2}}{R} \frac{\Gamma(\alpha-3 / 2)}{\Gamma(\alpha)} \sum_{n=-\infty}^{\infty} \frac{\Lambda^{2 \alpha-4}}{\left(\left(A_{4}+n / R\right)^{2}+m^{2}\right)^{\alpha-3 / 2}}
$$

At small values of $R$ we have

$$
\frac{1}{\epsilon}+\frac{\pi^{2}}{R} \frac{1}{\sqrt{A_{4}^{2}+m^{2}}}+F\left(R A_{4}, R m\right)
$$

where $F(x, y)$ is an analytic series vanishing as $x, y \rightarrow 0$.

13 We elaborate here on remarks in [37 38]. 
On the other hand, at large values of $R$ we find for the same integral:

$$
\frac{\pi^{2}}{\epsilon}-\pi^{2} \log \frac{m^{2}}{\Lambda^{2}}+2 \pi^{2} \sum_{n \neq 0} e^{2 \pi i n\left(A_{4} R\right)} K_{0}(2 \pi|n| m R)
$$

The physics of (6.39) is clear: The log term is the $4 \mathrm{~d}$ 1-loop effect of integrating out heavy charged particles in the low energy effective abelian theory. The Bessel functions from the nonzero modes decay as $\sim \frac{1}{2 \sqrt{|n| m R}} e^{-2 \pi|n| m R}$ and can be understood, from the $3 \mathrm{D}$ perspective, as instantons from particles of mass $m$ running in the $\mathbf{S}^{1}$ loop. As pointed out in [7], such quantum corrections are expected to renormalize the metric to a smooth hyperkähler metric on the moduli space. The expression (6.38) can then be understood as a modification

$$
\frac{8 \pi}{e_{3, I R}^{2}}=\frac{8 \pi}{e_{3, U V}^{2}}-16 \pi \sum_{i}(-1)^{\epsilon_{i}} Q_{i}^{2} \frac{\pi^{2}}{\sqrt{A_{4}^{2}+m^{2}}}
$$

Identifying $A_{4}^{2}+m^{2}$ with $\vec{\phi}^{2}$ we reproduce the 3D 1-loop result. Dualization of the photon then leads to a Taub-NUT metric with

$$
V \propto 1+\frac{M_{T N}}{r}
$$

Here $M_{T N}$ is the "Taub-NUT mass," and $r$ is the radial Euclidean distance in the standard representation of the TN space as a circle fibration over $\mathbb{R}^{3}[30]$.

Comparing (6.40) and (6.41) we see that there is a direct connection between the sign of the TN mass and the sign of the coefficient of the $4 \mathrm{D} \beta$-function. A negative mass $M_{T N}$ in $3 \mathrm{D}$ corresponds to an asymptotically free beta function in $4 \mathrm{D}$. This leads to an apparent paradox: The SW 3D target space is the Atiyah-Hitchin manifold $\mathcal{M}_{0}$ as $R \rightarrow 0$. The latter is well-known to be approximately a negative mass TN space for $r \rightarrow \infty$. How is this consistent with the concentration of the $u$-plane measure at $u= \pm 1$ for $R \rightarrow 0$ ? Indeed, when one examines the detailed map between $u$-plane coordinates (6.2) and coordinates $\sigma, \vec{\phi}$ on the Atiyah-Hitchin manifold (such as those used in (6.3)) one finds a complicated relation between "regions at infinity." In particular, regions of large $|\vec{\phi}|$ can sit over finite points on the $u$-plane. Since the effective theory near $u= \pm 1$ is IR free one might expect to see a positive mass TN metric. How is this possible !?

The way out of this confusion is to note that the $4 \mathrm{D}$ one-loop analysis in this regime is not very meaningful. In particular, the monopoles are very light. From (6.32) we see that in the relevant portion of the $u$-plane they have a mass of order $\left|a_{D}(u)\right| \lesssim e^{-\pi / R^{2}}$, and hence expansions such as (6.39) do not converge.

Clearly, there is much more to understand here, but we leave it at that, for the moment. 


\subsection{Possible implications for string duality}

The low energy dynamics of D-branes and M-branes gives a novel and powerful approach to investigating supersymmetric Yang-Mills theory [39]. Conversely, results on supersymmetric gauge theory will probably teach us important things about branes. Here we make a preliminary remark on a possible implication of the present results for brane physics.

In the gauge-theory-from-D/M-brane framework, the naive equivalence of DonaldsonWitten/Seiberg-Witten theory on $Y \times \mathbf{S}^{1}$ to Rozansky-Witten/Seiberg-Witten theory on $Y$ can be easily proved using standard string dualities. To do this one begins with the description of $d=4, \mathcal{N}=2$ theory as the low energy theory of an $M 5$ brane with two dimensions wrapped on the Seiberg-Witten curve [40]. In the IIA limit the configuration is described by parallel solitonic 5branes connected by D4-branes as in the Hanany-Witten setup [41]. If the solitonic 5branes wrap $Y \times \mathbf{S}^{1}$ we can apply $T$-duality along the $\mathbf{S}^{1}$, and then $S$-duality to obtain an effective 3D theory whose low energy dynamics is described by monopole moduli spaces, such as $\mathcal{M}_{0}$. Our computation shows that, at least for some quantities, like the partition function with supersymmetry preserving boundary conditions, the application of duality should be applied with care.

\section{Conclusions}

In this paper we investigated the Donaldson-Witten partition function $Z_{D W}$ on $Y \times \mathbf{S}^{1}$ for $b_{1}(Y)>0$. We have found some interesting relations to the torsion of $Y$, reinterpreting the result of Meng and Taubes from the physical point of view, and gaining some information on Floer homology.

Some very interesting questions remain open. One important circle of questions is related to the rational homology spheres with $b_{1}(Y)=0$. These present new challenges since, in evaluating $Z_{D W}$ one must integrate over the $u$-plane with a density involving oneloop determinants. Ironically, the actual $u$-plane integral turns out to be trivial and is just the volume of the fundamental domain of $\Gamma^{0}(4)$. However, this is more than compensated by the subtleties of the required one-loop graphs. We defer a discussion of this subject to another occasion.

We have also discussed some interesting subtleties in dimensional compactification of SYM. It would be nice to understand more deeply than we have done here the origin of the discrepancy between $Z_{D W}\left(Y \times \mathbf{S}^{1}\right)$ and $Z_{R W}(Y)$ for manifolds of $b_{1}(Y)=1$. A 
good understanding of the hyperkähler metric on $\mathcal{M}_{R}$ and the relation between regions at infinity in the $u$-plane and Atiyah-Hitchin descriptions would be very helpful.

Finally, our discussion has some potential applications in string duality, as mentioned in section 6.4.

\section{Acknowledgements}

We would like to thank P. Kronheimer, T. Li, M. Marcolli, G. Meng, V. Muñoz, N. Seiberg and B.L. Wang for very useful discussions and correspondence. We are specially indebted to E. Witten for many useful discussions and for his observations on a preliminary version of this paper. This work is supported by DOE grant DE-FG02-92ER40704. 


\section{References}

[1] E. Witten, "Topological Quantum Field Theory," Comm. Math. Phys. 117 (1988) 353.

[2] E. Witten, "Supersymmetric Yang-Mills theory on a four-manifold," hep-th/9403193; J. Math. Phys. 35 (1994) 5101.

[3] E. Witten, "Monopoles and four-manifolds," hep-th/9411102; Math. Res. Letters 1 (1994) 769.

[4] G. Moore and E. Witten, "Integration over the $u$-plane in Donaldson theory," hepth/9709193.

[5] M. Mariño and G. Moore, "Donaldson invariants for non-simply connected manifolds," hep-th/9804104.

[6] M. Mariño and G. Moore, "The Donaldson-Witten function for gauge groups of rank larger than one," hep-th/9802185.

[7] N. Seiberg and E. Witten, "Gauge dynamics and compactification to three dimensions," hep-th/9607163.

[8] L. Rozansky and E. Witten, "Hyperkähler geometry and invariants of three manifolds," hep-th/9612126.

[9] V.G. Turaev, "Reidemeister torsion in knot theory," Russ. Math. Surveys 41 (1986) 119.

[10] C. Lescop, Global surgery formula for the Casson-Walker invariant, Annals of Mathematical Studies, Princeton University Press, 1996.

[11] W.B.R. Lickorish, An introduction to knot theory, Springer, 1997.

[12] E. Witten, "Quantum Field Theory and the Jones Polynomial", Comm. Math. Phys. 121 (1989) 351.

[13] L. Rozansky, "A contribution of the trivial connection to the Jones polynomial and Witten's invariant of 3d manifolds, " Comm. Math. Phys. 175 (1996) 275.

[14] J. Morgan, T. Szabó and C.H. Taubes, "A product formula for the Seiberg-Witten invariants and the generalized Thom conjecture," J. Diff. Geometry 44 (1996) 706.

[15] G. Meng and C. Taubes, " $\underline{S W}=$ Milnor torsion", Math. Res. Lett. 3 (1996) 661.

[16] M. Marcolli, "Seiberg-Witten-Floer homology and Heegard splittings," Int. J. Math. 7 (1996) 671. M. Marcolli and B.-L. Wang, "Equivariant Seiberg-Witten-Floer homology," dg-ga/9606003.

[17] P. Kronheimer, "Embedded surfaces and gauge theory in three and four dimensions", preprint.

[18] A.L. Carey, B.L. Wang, R.B. Zhang and J. McCarthy, "Seiberg-Witten monopoles in three dimensions," Lett. Math. Phys. 39 (1997) 213. Y. Ohta, "Topological Field Theories associated with three-dimensional Seiberg-Witten monopoles," Int. J. Theor. Phys. 37 (1998) 925. 
[19] J.W. Milnor and J.D. Stasheff, Characteristic classes, Princeton University Press, 1974.

[20] C. Okonek and A. Teleman, "Seiberg-Witten invariants for manifolds with $b_{2}^{+}=1$, and the universal wall-crossing formula," alg-geom/9603003; Int. J. Math. 7 (1996) 811.

[21] T.J. Li and A. Liu, "General wall-crossing formula," Math. Res. Lett. 2 (1995) 797.

[22] A. Losev, N. Nekrasov, and S. Shatashvili, "Issues in topological gauge theory," hepth/9711108; "Testing Seiberg-Witten solution," hep-th/9801061.

[23] V. Muñoz, "Basic classes for four-manifolds not of simple type," math.DG/9811089.

[24] V. Muñoz, "Ring structure of the Floer cohomology of $\Sigma \times \mathbf{S}^{1}$," dg-ga/9710029.

[25] G. Thompson, "On the Generalized Casson Invariant," hep-th/9811199

[26] D. Kotschick, " $S O(3)$-invariants for manifolds with $b_{2}^{+}=1$," Proc. London Math. Society 63 (1991) 426.

[27] A. Floer, "Instanton homology and Dehn surgery," in The Floer memorial volume, Birkhäuser, 1994.

[28] V. Muñoz, "Fukaya-Floer homology of $\Sigma \times \mathbf{S}^{1}$ and applications," dg-ga/9804081.

[29] N. Seiberg, "IR Dynamics on Branes and Space-Time Geometry," hep-th/9606017; Phys.Lett. B384 (1996) 81-85

[30] T. Eguchi, P.B. Gilkey, and A.J. Hanson, "Gravitation, Gauge Theories, and Differential Geometry", Phys. Rep. 66(1980) 214.

[31] N. Dorey, V. V. Khoze, M. P. Mattis, D. Tong, S. Vandoren, "Instantons, ThreeDimensional Gauge Theory, and the Atiyah-Hitchin Manifold," hep-th/9703228; Nucl. Phys. B 502 (1997) 59.

[32] E. Witten, "Topology changing amplitudes in $(2+1)$ dimensional gravity, " Nucl. Phys. B 323 (1989) 113.

[33] M.F. Atiyah and L. Jeffrey, "Topological Lagrangians and cohomology," J. Geom. Phys. 7 (1990) 119.

[34] M. Blau and G. Thompson, " $\mathcal{N}=2$ topological gauge theory, the Euler characteristic of moduli spaces, and the Casson invariant," Comm. Math. Phys. 152 (1993) 41.

[35] R. Dijkgraaf and G. Moore, "Balanced Topological Field Theories," hep-th/9608169; Comm. Math. Phys. 185 (1997) 411.

[36] M. Bershadsky, S. Cecotti, H.Ooguri and C. Vafa, "Holomorphic anomalies in topological field theory," Nucl. Phys. B 405 (1993) 279.

[37] S. Shenker, "Another length scale in string theory?," hep-th/9509132.

[38] N. Seiberg and S. Shenker, "Hypermultiplet moduli space and string compactification to three dimensions," hep-th/9608086, Phys. Lett. B 388 (1996) 521.

[39] A. Giveon and D. Kutasov, "Brane Dynamics and Gauge Theory," hep-th/9802067

[40] E. Witten, "Solutions Of Four-Dimensional Field Theories Via M Theory," hepth/9703166; Nucl. Phys. B 500 (1997) 3.

[41] A. Hanany and E. Witten, "Type IIB Superstrings, BPS Monopoles, And ThreeDimensional Gauge Dynamics," hep-th/9611230; Nucl. Phys. B 492 (1997) 152. 